\title{
Consideration of various aspects in a drift study of MH370 debris
}

\author{
Oleksandr Nesterov ${ }^{1, \mathrm{a}}$ \\ ${ }^{1}$ independent consultant: Physial Oceanography \& Coastal Engineering, Dubai, UAE \\ anow at: DHI Water \& Environment (S) Pty Ltd., 2 Venture Drive, \# 18-18 Vision Exchange, \\ Singapore 608526, Singapore
}

Correspondence: Oleksandr Nesterov (oleksandr.nesterov@ gmail.com)

Received: 1 October 2017 - Discussion started: 4 December 2017

Revised: 16 April 2018 - Accepted: 25 April 2018 - Published: 4 June 2018

\begin{abstract}
On 7 March 2014, a Boeing 777-200ER aircraft operated by Malaysian Airlines as MH370 on the route from Kuala Lumpur to Beijing abruptly ceased all communications and disappeared with 239 people aboard, leaving its fate a mystery. The subsequent analysis of so-called satellite "handshakes" supplemented by military radar tracking has suggested that the aircraft ended up in the southern Indian Ocean. The eventual recovery of a number of fragments washed ashore in several countries has confirmed its crash. A number of drift studies were undertaken to assist in locating the crash site, mostly focusing either on the spatial distribution of the debris washed ashore or on the efficacy of the aerial search operation. A recent biochemical analysis of the barnacles attached to the flaperon (the first fragment found in La Réunion) has indicated that their growth likely began in water of $24^{\circ} \mathrm{C}$; then the temperature dropped to $18^{\circ} \mathrm{C}$, and then it rose up again to $25^{\circ} \mathrm{C}$. An attempt was made in the present study to take into consideration all these aspects. The analysis was conducted by means of numerical screening of 40 hypothetical locations of the crash site along the so-called seventh arc. Obtained results indicate the likelihood of the crash site to be located between 25.5 and $30.5^{\circ} \mathrm{S}$, with the segment from 28 to $30^{\circ} \mathrm{S}$ being the most promising.
\end{abstract}

\section{Introduction}

On 7 March 2014, approximately $40 \mathrm{~min}$ after the takeoff, a Boeing 777 aircraft (registration 9M-MRO) operated by Malaysian Airlines (MAS) as MH370 on the route from Kuala Lumpur (Malaysia) to Beijing (China), abruptly ceased all data and voice communications and disappeared with 239 people aboard, leaving investigators clueless about a possible reason for this. The subsequent analysis of socalled satellite "handshakes" (Ashton et al., 2015) supplemented by the primary radar tracking has suggested that the plane turned back, crossed the Malay Peninsula along the Malaysia-Thailand border, and then flew towards the Nicobar Islands in the Strait of Malacca, where it finally turned into the Indian Ocean, as detailed by the Australian Transport Safety Bureau (ATSB) (ATSB, 2014a, b) and the Ministry of Transport Malaysia (MTM, 2017). The eventual recovery of a number of 9M-MRO fragments, which were washed ashore in several countries, has confirmed the crash of the aircraft in the Indian Ocean. A total of 27 suspected and confirmed fragments were found according to the report published by the Malaysian Safety Investigation Team for MH370 (MSIT, 2017) on 27 March 2017: 1 in La Réunion, 2 in Mauritius, 1 in Rodrigues Island, 6 in Mozambique, 5 in South Africa, 1 in Tanzania, and 11 in Madagascar, with the latest find in January 2017.

Shortly after the disappearance, the Australian Government, whose geographical responsibility for rescue and recovery covers a region of the Indian Ocean where the terminus of 9M-MRO path could have been located according to the satellite data (Ashton et al., 2015), established the Joint Agency Coordination Centre (JAAC) (JAAC, 2014) to assist in the search operation. The Australian Maritime Safety Authority (AMSA, 2014), JAAC and ATSB have conducted an extensive aerial search operation, which lasted from 18 March to 27 April 2014, but failed to locate any debris related to MH370. Although some objects were spotted from the air, subsequent attempts to recover them were unsuccessful. After the expiration of the underwater locator beacon, a device emitting an acoustic signal to facilitate underwater search, the Australian Government commissioned an 
engineering company Fugro N.V., which specialises on marine and geotechnical surveys, to conduct a deep-water highresolution sonar survey of the seabed. The search domain assigned to Fugro N.V. was an elongated area in the southern Indian Ocean along the so-called seventh arc from approximately 36 to $39.5^{\circ} \mathrm{S}$, defined by the ATSB (2015), and then later refined by the Australian Defense Science and Technology Group (Davey et al., 2016). The seventh arc is a geometric curve on the Earth surface, all points of which are equidistant from the satellite, through which the last "handshake" was transmitted (Ashton et al., 2015). The actual seventh arc may slightly differ from the nominal arc due to the uncertainty in the altitude of the aircraft, as well as truncation and measurement errors in the data (e.g. ATSB, 2015; Davey et al., 2016). Despite such an unprecedented effort, the underwater search was unsuccessful, and it was finally called off in January 2017 (MTM, 2017).

A number of drift studies have been undertaken since then to assist in locating the crash site. Prior to the discovery of the flaperon in La Réunion on 29 July 2015, the studies focused on the analysis of the efficacy of the aerial search, as, for example, in García-Garrido et al. (2015). Later, after the flaperon and other 9M-MRO fragments were found, the mainstream approach shifted to the analysis of the probabilities of debris to reach specific locations by known dates starting from various origins along the seventh arc. Examples of such studies are as follows: the series of numerical and experimental studies undertaken by Griffin et al. (2017) at the Commonwealth Scientific and Industrial Research Organisation (CSIRO); the screening of 25 hypothetical locations along the seventh arc by Pattiaratchi and Wijeratne (2016) at the University of Western Australia; the numerical modelling conducted by Ormondt and Baart (2015) at Deltares; the study conducted by Maximenko et al. (2015) at the International Pacific Research Centre (IPRC); the study by Jansen et al. (2016) at the Euro-Mediterranean Center on Climate Change; the analysis of drifter trajectories obtained from National Oceanic and Atmospheric Administration's (NOAA) Global Drifter Program (GDP) in relation to MH370 debris by Trinanes et al. (2016). An alternative approach was based on the reverse drift modelling as in the studies conducted by the French Government meteorological agency Météo France (Daniel, 2016) and the GEOMAR Helmholtz Centre for Ocean Research Kiel (Durgadoo and Biastoch, 2015). The latter, however, did not account for wind forcing, which presumably explains the large difference in its conclusions compared to other studies.

There is ongoing disagreement between the conclusions of these studies with regard to the most likely origin of the debris at the seventh arc: the latest report published by CSIRO (Griffin et al., 2017) recommends a new search area at around $35^{\circ} \mathrm{S}$, backed by the earlier IPRC study ( 34 to $\left.37^{\circ} \mathrm{S}\right)$. In contrast, Pattiaratchi and Wijeratne (2016) have suggested the crash site to be more likely located between 28.3 and $33.2^{\circ} \mathrm{S}$, narrowing down earlier Jansen et al. (2016) estimates (be- tween 28 and $35^{\circ} \mathrm{S}$ ) and being also consistent with Ormondt and Baart (2015). Assuming zero drift angle of the flaperon, Daniel (2016) favours the location north of $25^{\circ} \mathrm{S}$ but south of $35^{\circ} \mathrm{S}$ if the drift angle was set to $18^{\circ}$ to the left with regard to wind at the leeway factor of $3.29 \%$ - both parameters experimentally established by the Direction générale de l'Armement (DGA). Griffin et al. (2017) disagrees with these parameters but confirms observed non-zero drift angles between 0 to $30^{\circ}$, explaining this effect by the longwise asymmetry of the flaperon.

An important feature of the fragment found in La Réunion are barnacles attached to it. Although it was not possible to establish their age, according to De Deckker (2017), who conducted biochemical analysis of the barnacles at the Australian National University, the start of their growth was in water of approximately $24^{\circ} \mathrm{C}$; then for some time the temperature ranged between 20 and $18^{\circ} \mathrm{C}$, and then it went up again to around $25^{\circ} \mathrm{C}$. This additional information has not been previously considered in the drift studies.

Consequently, this study comprises three major elements to assess the most likely origin of the debris: (1) the efficacy of the aerial search campaign, (2) ambient water temperatures at the flaperon, and (3) spatial distribution of debris washed ashore.

\section{Modelling}

A total of 40 hypothetical locations of the debris origin along the seventh arc were screened against the three selection criteria by means of forward particle tracking technique. The deterministic forcing of each particle in an ensemble was governed by the balance of water and air drag forces, magnitudes of which were assumed to be proportional to the squared relative (with respect to the particle) speeds of the ambient water and air respectively. Surface current velocities were sourced from the Hybrid Coordinate Ocean Model (HYCOM); wind data were sourced from the Global Data Assimilation System (GDAS), as detailed in Sect. 2.2.2. The stochastic component was modelled using the random walk technique (e.g. Al-Rabeh et al., 2000; DHI, 2009; Jansen et al., 2016). Numerical integration was performed in the geocentric Cartesian coordinate system.

All the particles in an ensemble were "released" from a single starting point at the seventh arc. Four models with regard to the leeway and drift angle properties of particles were considered. After individual particle tracks were obtained, they were supplemented by respective sea surface temperatures (SSTs) extracted from the publicly available archives. A subsequent analysis was undertaken in a statistical manner to (1) estimate the maximum ensemble coverages during the aerial search, (2) assess the percentage of particles in ensembles which could have reached La Réunion by 29 July 2015 and could have been subjected to the temperature variation matching the results of the barnacle analysis, and (3) com- 


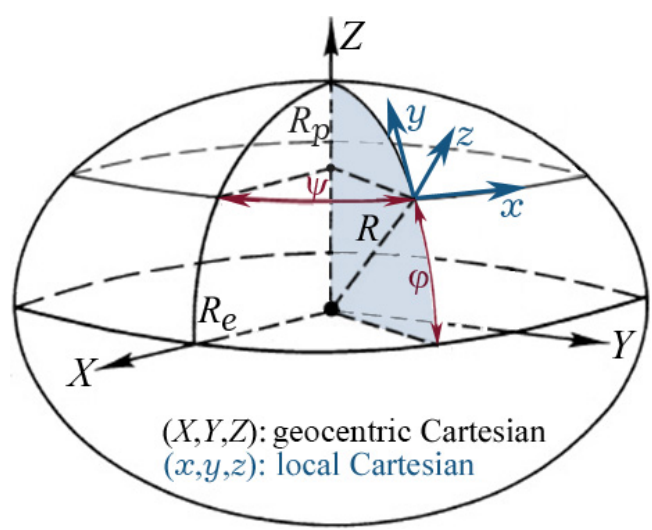

Figure 1. Local and geocentric Cartesian coordinate systems.

pare the spatial distribution of particles washed ashore with the locations where MH370 fragments were found.

\subsection{Model description}

\subsubsection{Assumptions}

In the context of this study it was assumed that a particle was subjected to the drag forces induced by water and wind. Particles were assumed to be non-inertial. Impacts of the Coriolis force, Stokes drift, waves, decay, and sinking were neglected (e.g. Kraus, 1972; Al-Rabeh et al., 2000). In the local coordinate system $(x, y)$, where the axes $x$ and $y$ correspond to the local west-to-east and south-to-north directions respectively (Fig. 1), the location $\left(x_{i}, y_{i}\right)$ of the $i$ th particle was described by Langevin's equation (DHI, 2009):

$\frac{\mathrm{d} x_{i}}{\mathrm{~d} t}=\bar{u}_{i}+D\left(t, x_{i}, y_{i}\right) \zeta, \frac{\mathrm{d} y_{i}}{\mathrm{~d} t}=\bar{v}_{i}+D\left(t, x_{i}, y_{i}\right) \xi$,

where $\left(\bar{u}_{i}, \bar{v}_{i}\right)$ is the average (deterministic) velocity of the $i$ th particle in the local coordinate system, $D$ is the turbulent diffusion term, and $\zeta, \xi$ are the random numbers, as detailed in Sect. 2.1.3 through 2.1.5.

\subsubsection{Coordinate systems}

On the one hand, the velocity of a particle and random walk are formulated in the local coordinate system, where the axis $z$ is normal to the Earth surface. On the other hand, the large study domain dictates the necessity to properly take into consideration the Earth curvature. To perform numerical integration of the governing equations in the geocentric Cartesian coordinate system $(X, Y, Z)$ depicted in Fig. 1, where the Earth surface is approximated by WGS 84 ellipsoid with the polar and equatorial axes radii $R_{\mathrm{p}}=6356752$ and $R_{\mathrm{e}}=6378137 \mathrm{~m}$ respectively, the transformations of coordinates and velocity vectors are required.
The Cartesian coordinates $X, Y$, and $Z$ of a point on the surface of the ellipsoid described by the longitude $\psi$ and geocentric latitude $\varphi$ can be formulated as

$X=R \cos \varphi \cos \psi, Y=R \cos \varphi \sin \psi, Z=R \sin \varphi$,

where

$$
R=\frac{R_{\mathrm{e}} R_{\mathrm{p}}}{\sqrt{\left(R_{\mathrm{p}} \cos \varphi\right)^{2}+\left(R_{\mathrm{e}} \sin \varphi\right)^{2}}}
$$

is the distance between this point and the centre of the ellipsoid, as follows from the ellipse equation

$\frac{(R \cos \varphi)^{2}}{R_{\mathrm{e}}^{2}}+\frac{(R \sin \varphi)^{2}}{R_{\mathrm{p}}^{2}}=1$.

It should be noted that the backward transformation is required to extract and interpolate surface current and wind data at a given location. Respective trigonometric transformations involve solving a fourth-degree polynomial equation.

The unit vectors $\overrightarrow{\boldsymbol{L}}, \overrightarrow{\boldsymbol{M}}$, and $\overrightarrow{\boldsymbol{N}}$, which define the directions of the axes $x, y$, and $z$ of the local coordinate system (Fig. 1), are introduced to obtain velocity components in the geocentric system. The outward unit vector $\vec{N}=\left\{N_{X}, N_{Y}, N_{Z},\right\}$ normal to the surface of the ellipsoid is formulated according to Korn and Korn (1968):

$\vec{N}=\frac{\left\{\frac{\partial X}{\partial \psi}, \frac{\partial Y}{\partial \psi}, \frac{\partial Z}{\partial \psi}\right\} \times\left\{\frac{\partial X}{\partial \varphi}, \frac{\partial Y}{\partial \varphi}, \frac{\partial Z}{\partial \varphi}\right\}}{\left|\left\{\frac{\partial X}{\partial \psi}, \frac{\partial Y}{\partial \psi}, \frac{\partial Z}{\partial \psi}\right\} \times\left\{\frac{\partial X}{\partial \varphi}, \frac{\partial Y}{\partial \varphi}, \frac{\partial Z}{\partial \varphi}\right\}\right|}=$

$N_{X}=\frac{1}{\sqrt{1+\mu^{2}}}(\cos \varphi-\mu \sin \varphi) \cos \psi$,

$N_{Y}=\frac{1}{\sqrt{1+\mu^{2}}}(\cos \varphi-\mu \sin \varphi) \sin \psi$,

$N_{Z}=\frac{1}{\sqrt{1+\mu^{2}}}(\sin \varphi+\mu \cos \varphi)$,

where $\mu=R^{2} \frac{R_{\mathrm{e}}^{2}-R_{\mathrm{p}}^{2}}{R_{\mathrm{e}}^{2} R_{\mathrm{p}}^{2}} \cos \varphi \sin \varphi$.

The direction of the axis $x$ is defined by the unit vector $\overrightarrow{\boldsymbol{L}}$ :

$\overrightarrow{\boldsymbol{L}}=\left\{L_{X}, L_{Y}, L_{Z}\right\}=\{-\sin \psi, \cos \psi, 0\}$.

Equations (3) and (4) allow expressing the unit vector $\overrightarrow{\boldsymbol{M}}=\left\{M_{X}, M_{Y}, M_{Z}\right\}$ collinear with the axis $y$ in the form of the vector product $\overrightarrow{\boldsymbol{M}}=\overrightarrow{\boldsymbol{N}} \times \overrightarrow{\boldsymbol{L}}$, so that its components are

$M_{X}=-\frac{1}{\sqrt{1+\mu^{2}}}(\sin \varphi+\mu \cos \varphi) \cos \psi$, 


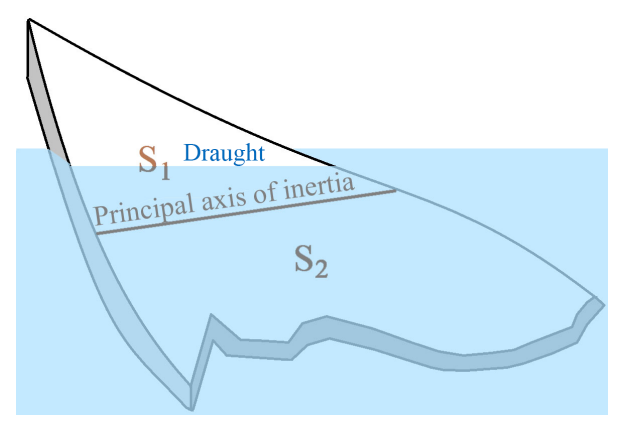

Figure 2. Schematic representation of a thin floating object.

$$
\begin{aligned}
& M_{Y}=-\frac{1}{\sqrt{1+\mu^{2}}}(\sin \varphi+\mu \cos \varphi) \sin \psi, \\
& M_{Z}=\frac{1}{\sqrt{1+\mu^{2}}}(\cos \varphi-\mu \sin \varphi) .
\end{aligned}
$$

Therefore, a velocity vector, the components of which are $\{\bar{u}, \bar{v}, 0\}$ in the local system, has the following components in the geocentric Cartesian system:

$$
\begin{aligned}
& \bar{U}=-\bar{u} \sin \psi-\bar{v} \frac{1}{\sqrt{1+\mu^{2}}}(\sin \varphi+\mu \cos \varphi) \cos \psi, \\
& \bar{V}=\bar{u} \cos \psi-\bar{v} \frac{1}{\sqrt{1+\mu^{2}}}(\sin \varphi+\mu \cos \varphi) \sin \psi, \\
& \bar{W}=\quad \bar{v} \frac{1}{\sqrt{1+\mu^{2}}}(\cos \varphi-\mu \sin \varphi) .
\end{aligned}
$$

Hence, Langevin's Eq. (1) can be formulated in the geocentric Cartesian system as follows:

$$
\left\{\begin{aligned}
\frac{\mathrm{d} X_{i}}{\mathrm{~d} t} & =\bar{U}\left(\bar{u}_{i}, \bar{v}_{i}, \psi_{i}, \varphi_{i}\right)+D\left(L_{X} \zeta+M_{X} \xi\right), \\
\frac{\mathrm{d} Y_{i}}{\mathrm{~d} t} & =\bar{V}\left(\bar{u}_{i}, \bar{v}_{i}, \psi_{i}, \varphi_{i}\right)+D\left(L_{Y} \zeta+M_{Y} \xi\right), \\
\frac{\mathrm{d} Z_{i}}{\mathrm{~d} t} & =\bar{W}\left(\bar{u}_{i}, \bar{v}_{i}, \psi_{i}, \varphi_{i}\right)+D\left(L_{Z} \zeta+M_{Z} \xi\right),
\end{aligned}\right.
$$

where the relations between the longitude $\psi_{i}$ and geocentric latitude $\varphi_{i}$ of particle's location and its geocentric Cartesian coordinates $X_{i}, Y_{i}, Z_{i}$ are given by Eq. (2); the transformation of the velocity components is given by Eq. (6); and the local velocity components $\bar{u}_{i}$, and $\bar{v}_{i}$ are defined from the balance of the deterministic forces.

\subsubsection{Deterministic terms}

The mathematical description of the dynamics of a floating object is not a trivial problem due to the variety of processes and phenomena in a near-surface layer, such as surface waves, Stokes drift, flow-object interaction, buoyancy, and stratification (e.g. Kraus, 1972). Such an object is subjected to the dynamic pressure and shear stress forces due to the action of the water and air; its steady-state orientation depends on its buoyancy characteristics and the moments of forces around its principal axes of inertia. Denoting $\overrightarrow{\overrightarrow{\boldsymbol{u}}}_{i}=\left(\bar{u}_{i}, \bar{v}_{i}\right)$ the deterministic velocity of the $i$ th particle representing a floating object in the local coordinate system and neglecting the Coriolis force, its motion in the local horizontal plane is described by the following equation:

$m_{i} \frac{\mathrm{d} \overrightarrow{\boldsymbol{u}}_{i}}{\mathrm{~d} t}=\overrightarrow{\overline{\boldsymbol{F}}_{\mathrm{w}, i}}+\overrightarrow{\overline{\boldsymbol{F}}_{\mathrm{a}, i}}$

where $m_{i}$ is the mass of the object and $\overrightarrow{\overline{\boldsymbol{F}}_{\mathrm{w}, i}}$ and $\overrightarrow{\overline{\boldsymbol{F}}_{\mathrm{a}, i}}$ are the average forces caused respectively by water and air flows around the object. The same formulation of these forces as applied in Daniel et al. (2002) and Breivik et al. (2011) to study drift of ship containers was adopted in this study, although justification for thin nearly horizontally floating objects (Fig. 2) could be slightly different. According to the theory of a turbulent boundary layer (e.g. Kraus, 1972; Gandin et al., 1955), vertical velocity profiles of the water and air exhibit a logarithmic dependence on the distance from the surface

$U(z)=\frac{u_{*}}{\kappa} \ln \frac{|z|}{z_{0}}$,

where $u_{*}$ is the friction velocity, $\kappa=0.41$ is von Kármán's constant, and $z_{0}$ is roughness, where the surface is assumed to be non-moving at $z=0$. The turbulent shear stresses $\tau_{\{\mathrm{w}, \mathrm{a}\}}=\rho_{\{\mathrm{w}, \mathrm{a}\}} u_{*\{w, a\}}^{2}$, where $\rho_{\{\mathrm{w}, \mathrm{a}\}}$ is the density of the water or air, remain constant through this layer. Hence, the shear stresses acting on the top and bottom surfaces of a thin floating object can be considered proportional to $\left|\overrightarrow{\overrightarrow{\boldsymbol{u}}_{\mathrm{w}}}-\overrightarrow{\overrightarrow{\boldsymbol{u}}}\right|^{2}$ and $\left|\overrightarrow{\boldsymbol{u}_{\mathrm{a}}}-\overrightarrow{\overrightarrow{\boldsymbol{u}}}\right|^{2}$, where $\overrightarrow{\overline{\boldsymbol{u}}}_{\mathrm{w}}=\overrightarrow{\overline{\boldsymbol{u}}}_{\mathrm{w}}\left(x_{i}, y_{i}, t\right)$ and $\overrightarrow{\overline{\boldsymbol{u}}}_{\mathrm{a}}=\overrightarrow{\overline{\boldsymbol{u}}}_{\mathrm{a}}\left(x_{i}, y_{i}, t\right)$ are the current and wind velocities at the location of the object at a certain reference depth and height (the typical reference height for wind is $10 \mathrm{~m}$ above the surface). The dynamic pressures can also be considered proportional to the squared relative velocities of water and air but at some other representative distances. Bearing in mind the logarithmic velocity profile, the latter would also be proportional to $\left|\overrightarrow{\overrightarrow{\boldsymbol{u}}_{\mathrm{w}}}-\overrightarrow{\overrightarrow{\boldsymbol{u}}}\right|^{2}$ and $\left|\overrightarrow{\boldsymbol{u}}_{\mathrm{a}}-\overrightarrow{\boldsymbol{u}}\right|^{2}$ respectively. Therefore, if the drag forces are collinear with the respective relative velocities of the water and air, the same formulation as in Daniel et al. (2002) would also be applicable for thin objects:

$$
\begin{aligned}
& {\overrightarrow{\boldsymbol{F}_{\mathrm{w}, i}}}=\frac{1}{2} C_{\mathrm{Dw}, i} S_{\mathrm{w}, i} \rho_{\mathrm{w}}\left|\overrightarrow{\overline{\boldsymbol{u}}}_{\mathrm{w}}-\overrightarrow{\overline{\boldsymbol{u}}}_{i}\right|\left(\overrightarrow{\overline{\boldsymbol{u}}}_{\mathrm{w}}-\overrightarrow{\overline{\boldsymbol{u}}}_{i}\right), \\
& {\overrightarrow{\boldsymbol{F}_{\mathrm{a}, i}}}=\frac{1}{2} C_{\mathrm{Da}, i} S_{\mathrm{a}, i} \rho_{\mathrm{a}}\left|\overrightarrow{\overline{\boldsymbol{u}}}_{\mathrm{a}}-\overrightarrow{\overline{\boldsymbol{u}}}_{i}\right|\left(\overrightarrow{\overline{\boldsymbol{u}}}_{\mathrm{a}}-\overrightarrow{\overline{\boldsymbol{u}}}_{i}\right),
\end{aligned}
$$

where $C_{\mathrm{Dw}, i}$ and $S_{\mathrm{w}, i}$ are the water drag coefficient and corresponding reference area of the submerged part of the ob- 
ject, $C_{\mathrm{Da}, i}$ and $S_{\mathrm{a}, i}$ are the air drag coefficient and corresponding reference area of the part exposed to the air, $\rho_{\mathrm{w}}$ and $\rho_{\mathrm{a}}$ are the water and air densities. Furthermore, Breivik et al. (2011) argued that wave drift forces on small objects (less than $30 \mathrm{~m}$ ) decay rapidly, and they can be neglected compared to wind forces when the wave length is more than 6 times the dimensions of a floating object.

It should be noted that composite materials are usually light-weight structures. For example, the recovered flaperon is approximately $1.6 \mathrm{~m} \times 2.4 \mathrm{~m} \times 0.25 \mathrm{~m}$ in size and $50 \mathrm{~kg}$ in weight. Bearing in mind that the typical values of the minimum drag coefficient $C_{\mathrm{Da}}$ for airfoils are in the range of 0.02 to 0.05 with respect to their surface areas, it is easy to show that the drag forces acting on such a fragment of the aircraft would normally be much larger compared to the inertial term in Eq. (8), and hence the latter can be approximated by the balance equation: $\overrightarrow{\bar{F}}_{\mathrm{w}, i}=-\overrightarrow{\overline{\boldsymbol{F}}}_{\mathrm{a}, i}$. Using Eq. (9), the latter yields

$$
\begin{aligned}
& \frac{1}{2} C_{\mathrm{Dw}, i} S_{\mathrm{w}, i} \rho_{\mathrm{w}}\left|\overrightarrow{\overline{\boldsymbol{u}}}_{i}-\overrightarrow{\overline{\boldsymbol{u}}}_{\mathrm{w}}\left(x_{i}, y_{i}, t\right)\right|\left(\overrightarrow{\overline{\boldsymbol{u}}}_{i}-\overrightarrow{\overline{\boldsymbol{u}}}_{\mathrm{w}}\left(x_{i}, y_{i}, t\right)\right)= \\
& -\frac{1}{2} C_{\mathrm{Da}, i} S_{\mathrm{a}, i} \rho_{\mathrm{a}}\left|\overrightarrow{\overline{\boldsymbol{u}}}_{i}-\overrightarrow{\overline{\boldsymbol{u}}}_{\mathrm{a}}\left(x_{i}, y_{i}, t\right)\right|\left(\overrightarrow{\overline{\boldsymbol{u}}}_{i}-\overrightarrow{\boldsymbol{u}}_{\mathrm{a}}\left(x_{i}, y_{i}, t\right)\right) .
\end{aligned}
$$

It is easy to see that the solution of Eq. (10) is

$$
\overrightarrow{\overrightarrow{\boldsymbol{u}}_{i}}=\left(1-\alpha_{i}\right) \overrightarrow{\overline{\boldsymbol{u}}}_{\mathrm{w}}\left(x_{i}, y_{i}, t\right)+\alpha_{i} \overrightarrow{\overrightarrow{\boldsymbol{u}}}_{\mathrm{a}}\left(x_{i}, y_{i}, t\right),
$$

where the scalar $\alpha_{i}$ (leeway factor) is

$\alpha_{i}=\frac{\sqrt{C_{\mathrm{Da}, i} S_{\mathrm{a}, i} \rho_{\mathrm{a}}}}{\sqrt{C_{\mathrm{Da}, i} S_{\mathrm{a}, i} \rho_{\mathrm{a}}}+\sqrt{C_{\mathrm{Dw}, i} S_{\mathrm{w}, i} \rho_{\mathrm{w}}}}$.

For a thin horizontally floating object, which has equal areas of the surface exposed to seawater and air $\left(S_{\mathrm{a}}=S_{\mathrm{w}}\right)$ and which is characterised by equal drag coefficients $C_{\mathrm{Da}}=C_{\mathrm{Dw}}$, the leeway factor is $\alpha \approx 3.33 \%$. This theoretical value is in a good agreement with the experimental data for the flaperon $(3.29 \%)$ estimated by the DGA in the hydrodynamic engineering test facility centre in Toulouse (Daniel, 2016). For a $70 \%$-submerged cube, this formulation yields $\alpha=2.2 \%$ assuming the dominance of the dynamic pressure acting on the lateral faces and being also in a good agreement with the experimental factor of $2 \%$ (Breivik et al., 2011).

In two of the four considered models (Sect. 2.2), where the force induced by relative wind was not collinear with its direction, a modified formulation was used instead of Eq. (11):

$\overrightarrow{\overrightarrow{\boldsymbol{u}}_{i}}=\left(1-\alpha_{i}\right) \overrightarrow{\overrightarrow{\boldsymbol{u}}_{\mathrm{w}}}+\alpha_{i}\left(\begin{array}{rr}\cos \theta & -\sin \theta \\ \sin \theta & \cos \theta\end{array}\right) \overrightarrow{\overrightarrow{\boldsymbol{u}}}_{\mathrm{a}}$,

where $\theta$ is the drift angle and is positive counter-clockwise.

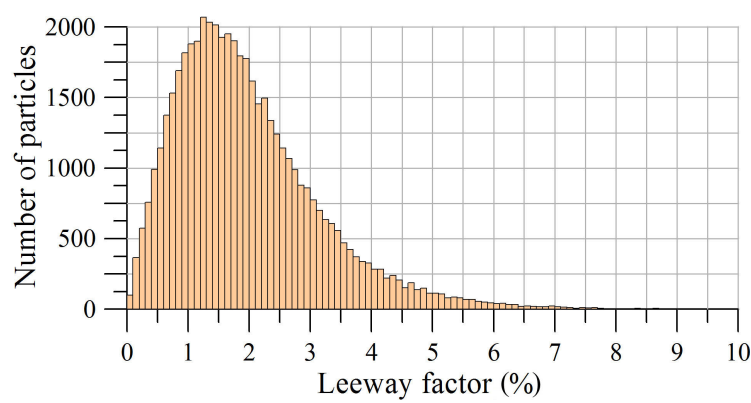

Figure 3. Assumed distribution of leeway factors across an ensemble (constant in time for an individual particle).

\subsubsection{Random leeway factor model}

In contrast to earlier studies (e.g. Daniel, 2016; Pattiaratchi and Wijeratne, 2016; Griffin et al., 2017), an attempt was made in this work to take into consideration the variety of leeway factors, which describe random shapes and flotation characteristics of individual fragments generated by the crash. For this purpose, in one of the four considered types of ensembles (Sect. 2.2) particles were described by random leeway factors. It was assumed that these particles represented partially submerged thin objects of irregular shapes floating in slightly tilted orientations (Fig. 2). For the sake of simplification, it was assumed that the drag coefficients for the air and water are equal $\left(C_{\mathrm{Da}, i}=C_{\mathrm{Ds}, i}\right)$. Then, according to Eq. (12), only the knowledge of the ratio of respective areas $k=S_{\mathrm{a}, i} / S_{\mathrm{w}, i}$ is required to estimate the leeway factor:

$\alpha_{i}=\frac{\sqrt{\rho_{\mathrm{a}} / \rho_{\mathrm{w}}} \sqrt{k_{i}}}{1+\sqrt{\rho_{\mathrm{a}} / \rho_{\mathrm{w}}} \sqrt{k_{i}}}$.

In the context of this study it was assumed that dimensions of individual objects are log-normally distributed. Furthermore, it was assumed that the principal axis of inertia of the object splits it into two parts, areas of which are also lognormally distributed, so that

$k_{i}=\frac{\gamma_{i} S_{i, 1}}{S_{i, 2}+\left(1.0-\gamma_{i}\right) S_{i, 1}}$,

where $\left\{\ln S_{i, 1}, \ln S_{i, 2}\right\} \in \mathcal{N}\left(\mu, \sigma^{2}\right)$ and $\gamma \in[0,1]$ is an independent random parameter to account for the draught of the object $(0-$ fully submerged; 1 - the centre of gravity is at the water surface). Hence the logarithm of the ratio $\ln \left(S_{1} / S_{2}\right)=\ln S_{1}-\ln S_{2} \in \mathcal{N}\left(0,2 \sigma^{2}\right)$ is also normally distributed, with a mean of 0 . Here the property was used that the sum of two independent normally distributed random variables is also normally distributed, with its mean being the sum of the means and its variance being the sum of the two variances (Eisenberg and Sullivan, 2008). Hence, the ratio $S_{1} / S_{2}$ is log-normally distributed. The modelling was performed assuming $\ln \left(S_{1} / S_{2}\right) \in \mathcal{N}(0,1)$. The resulting distribution of the leeway factors of particles in an ensemble in 
this class of simulations (hereafter referred to as the "random leeway" model) is depicted in Fig. 3. A random leeway factor assigned to a particle in ensemble was constant in time.

\subsubsection{Numerical realisation and random walk}

The whole integration interval was split into time steps of $\Delta t=15$ min. Similarly to the DHI (2009) particle tracking model, the integration of the system of equations (Eq. 7) for each particle over the time step $\Delta t$ was comprised of the two stages, namely the deterministic and the stochastic one:

$\overrightarrow{\boldsymbol{X}}_{i}(t+\Delta t)=\overrightarrow{\boldsymbol{X}}_{i}(t)+\int_{t}^{t+\Delta t} \overrightarrow{\overrightarrow{\boldsymbol{U}}}\left(\bar{u}_{i}, \bar{v}_{i}, \psi_{i}, \varphi_{i}\right) \mathrm{d} t$,
$\overrightarrow{\boldsymbol{X}}_{i}(t+\Delta t)=\overrightarrow{\overline{\boldsymbol{X}}}_{i}(t+\Delta t)+\overrightarrow{\boldsymbol{\delta}}_{i}(\ldots)$,

where $\overrightarrow{\boldsymbol{X}}_{i}=\left\{X_{i}, Y_{i}, Z_{i}\right\}$ is the location of the $i$ th particle; $\overrightarrow{\bar{X}}_{i}$ is the intermediate location prior to the superposition of the random displacement $\overrightarrow{\boldsymbol{\delta}_{i}}(\ldots) ; \overrightarrow{\vec{U}}\left(\bar{u}_{i}, \bar{v}_{i}, \psi_{i}, \varphi_{i}\right)=$ $\{\bar{U}(\ldots), \bar{V}(\ldots), \bar{W}(\ldots)\}$ is the velocity of the particle. Unlike the DHI (2009) model, which uses the first-order discretisation method to integrate deterministic terms, the fifthand sixth-order Runge-Kutta method was used in this work utilising FORTRAN libraries to ensure that particles remain on the ellipsoid's surface with sufficient accuracy. During the integration, input current and wind data were bilinearly interpolated in space and linearly in time.

The vector $\overrightarrow{\boldsymbol{\delta}_{i}}(\ldots)$ corresponds to a numerical solution for the diffusivity term of the Langevin Eq. (1). It was treated as a random displacement in the $x y$ plane locally tangential to ellipsoid's surface. Its components $\delta_{x}$ and $\delta_{y}$ in the local coordinate system are the random values from the trimmed two-dimensional Gaussian distribution $\mathcal{N}_{2}$ :

$\delta_{x}=\sigma_{L} \zeta, \quad \delta_{y}=\sigma_{L} \xi$,

where $\{\zeta, \xi\} \in \mathcal{N}_{2}(0,1)$ are the random numbers and the standard deviation of the turbulent dispersion $\sigma_{L}=\sqrt{2 D \Delta t}$ is assumed to be a function of the horizontal eddy diffusivity coefficient $D=D\left(t, \psi_{i}, \varphi_{i}\right)$. Such a relation between $D$ and $\sigma_{L}$ was first established by A. Einstein in 1905, who studied diffusion associated with Brownian motion, and since then it was adopted in a variety of random walk models (e.g. DHI, 2009; Jansen et al., 2016). In this study trimming was imposed to discard values $\delta_{x}$ and $\delta_{y}$ that resulted in displacements exceeding $10 \mathrm{~km}$ distance over the time step $\Delta t$. If this criterion was violated, the next pair of random values $\delta_{x}$ and $\delta_{y}$ was computed. The distance of $10 \mathrm{~km}$ was selected as the representative resolution of the ocean circulation model HY$\mathrm{COM}$, used as a source of the surface current velocity data (see Sect. 2.2.2).
In contrast to Jansen et al. (2016), who applied the constant eddy diffusivity coefficient $D=2 \mathrm{~m} \mathrm{~s}^{-1}$, in this work $D$ was computed according to the well-known Smagorinsky (1963) parameterisation, applied in various ocean circulation models (e.g. Blumberg and Mellor, 1987; DHI, 2009):

$D=\kappa \Delta x \Delta y \sqrt{\left(\frac{\partial u}{\partial x}\right)^{2}+\frac{1}{2}\left(\frac{\partial u}{\partial y}+\frac{\partial v}{\partial x}\right)^{2}+\left(\frac{\partial v}{\partial y}\right)^{2}}$,

where $\kappa=0.1$ is the constant coefficient (a typical range is from 0.1 to 0.2 according to Blumberg and Mellor, 1987), and $\Delta x$ and $\Delta y$ are the horizontal dimensions of the numerical grid cell, applied for the discretisation of the velocity field. A discrete approximation of Eq. (16) was used to estimate the eddy diffusivity coefficient $D$, with subsequent bilinear interpolation in space and linear interpolation in time at the actual locations of particles.

In the geocentric Cartesian coordinate system the random displacement translates into the $3-\mathrm{D}$ vector $\overrightarrow{\boldsymbol{\delta}_{i}}=$ $\left\{\delta X_{i}, \delta Y_{i}, \delta Z_{i}\right\}$, components of which are

$\delta X_{i}=L_{X} \delta_{x}+M_{X} \delta_{y}$
$\delta Y_{i}=L_{Y} \delta_{x}+M_{Y} \delta_{y}$
$\delta Z_{i}=L_{Z} \delta_{x}+M_{Z} \delta_{y}$

Such a displacement in the tangential plane causes a particle to move away from the surface of the ellipsoid. However, due to the imposed limitation on the distance, the elevation of the particle does not exceed $8 \mathrm{~m}$ after superposition of the random walk. Thus, particle elevations were forced to zero after applying the random walk procedure at each integration time step, while preserving longitudes and latitude.

A Box and Muller (1958) transform was used to obtain a pair of pseudo-random numbers $\{\zeta, \xi\} \in \mathcal{N}_{2}(0,1)$ :

$\zeta=\sqrt{-2 \ln \tau} \cos (2 \pi \omega), \quad \xi=\sqrt{-2 \ln \tau} \sin (2 \pi \omega)$,

where $\tau$ and $\omega$ are the two pseudo-random numbers from the interval $(0,1]$. To obtain $\tau$ and $\omega$, the use was made of the generator developed by Marsaglia and Zaman (1987), claimed to have the period of $2^{144}$.

Close to a shore, flow velocity was linearly interpolated between zero and velocity in the adjacent cell of the numerical grid of HYCOM. If a particle moved onshore as a result of wind action or random walk, all the subsequent forcing acting on such a particle was nullified, so that it remained at the location where it beached. No specific properties of the shores (e.g. type, slope) or contributing factors such as waves, tides, or storm surges, were considered.

It is worth noting that the transformations between the longitude, latitude, and geocentric Cartesian coordinates were performed using extended precision accuracy ( $80 \mathrm{bits}$ ). Con- 
Table 1. Longitudes and latitudes of the origins preselected for screening

\begin{tabular}{lccccccccccc}
\hline No. & Long, ${ }^{\circ} \mathrm{E}$ & Lat, ${ }^{\circ} \mathrm{S}$ & No. & Long, ${ }^{\circ} \mathrm{E}$ & $\mathrm{Lat},{ }^{\circ} \mathrm{S}$ & No. & Long, ${ }^{\circ} \mathrm{E}$ & Lat, $^{\circ} \mathrm{S}$ & No. & Long, ${ }^{\circ} \mathrm{E}$ & $\mathrm{Lat},{ }^{\circ} \mathrm{S}$ \\
\hline 1 & 85.72 & 39.17 & 11 & 92.29 & 35.29 & 21 & 98.07 & 30.03 & 31 & 102.64 & 23.69 \\
2 & 86.43 & 38.83 & 12 & 92.91 & 34.82 & 22 & 98.59 & 29.44 & 32 & 103.02 & 23.00 \\
3 & 87.13 & 38.48 & 13 & 93.53 & 34.34 & 23 & 99.09 & 28.84 & 33 & 103.39 & 22.31 \\
4 & 87.82 & 38.11 & 14 & 94.14 & 33.84 & 24 & 99.58 & 28.23 & 34 & 103.75 & 21.62 \\
5 & 88.51 & 37.73 & 15 & 94.73 & 33.33 & 25 & 100.05 & 27.61 & 35 & 104.18 & 20.74 \\
6 & 89.01 & 37.44 & 16 & 95.32 & 32.81 & 26 & 100.52 & 26.98 & 36 & 104.50 & 20.03 \\
7 & 89.69 & 37.03 & 17 & 95.89 & 32.28 & 27 & 100.97 & 26.34 & 37 & 104.82 & 19.31 \\
8 & 90.35 & 36.62 & 18 & 96.46 & 31.74 & 28 & 101.40 & 25.69 & 38 & 105.20 & 18.41 \\
9 & 91.00 & 36.19 & 19 & 97.01 & 31.18 & 29 & 101.83 & 25.03 & 39 & 105.48 & 17.68 \\
10 & 91.65 & 35.74 & 20 & 97.55 & 30.61 & 30 & 102.24 & 24.36 & 40 & 105.75 & 16.94 \\
\hline
\end{tabular}

ducted tests have shown that the maximum errors in the elevation of a particle, which resulted from a single forwardbackward conversion of the coordinates, were of the order of $0.5 \mathrm{~mm}$ using the extended precision compared to $0.5 \mathrm{~m}$ using the double-precision arithmetic.

\subsection{Model set-up}

Four models with respect to the leeway factor and drift angle of particles in an ensemble were considered:

- a leeway factor of $3.29 \%$; zero drift angle;

- a leeway factor of $3.29 \%$; drift angle of $18^{\circ}$ to the left;

- a leeway factor of $2.76 \%$; drift angle of $32^{\circ}$ to the left;

- random leeway factor; zero drift angle.

All the ensembles released at various locations were identical with respect to the properties of particles in these ensembles. Each ensemble comprised 50000 particles, the same as in Pattiaratchi and Wijeratne (2016). The second and third models focused specifically on the flaperon path: respective settings corresponded to the flotation characteristics established by the DGA (Daniel, 2016). The fourth model aimed to achieve a more realistic representation of the flotation characteristics of the debris generated by the crash by assigning random leeway factors (fixed in time) to the particles of an ensemble; all the 40 ensembles (Sect. 2.2.1) were identical in terms of the particles they were comprised of.

\subsubsection{Screened locations}

The study domain extended from 20 to $140^{\circ} \mathrm{E}, 55^{\circ} \mathrm{S}$ to $15^{\circ} \mathrm{N}$. Integration was performed from 8 March 2014 to 31 December 2016 inclusive. The locations of the 40 hypothetical debris origins are summarised in Table 1 and indicated in Fig. 4. The coordinates of these locations were estimated from the burst time offset (ATSB, 2015) of the last "handshake" 00:19, assuming that the aircraft was at the altitude of $10 \mathrm{~km}$.

\subsubsection{Model forcing and SST data}

The following datasets were used in this study for the model forcing and temperature analysis:

- Surface currents were extracted from the Hybrid Coordinate Ocean Model, a data-assimilative isopycnal-sigma-pressure coordinate ocean circulation model (Chassignet et al., 2007). Spatial resolution: $0.08^{\circ} \times 0.08^{\circ}$; temporal resolution: daily. The HYCOM consortium is a multiinstitutional effort sponsored by the National Ocean Partnership Program (https://hycom.org/, last access: 17 May 2018); data are available at ftp: //ftp.hycom.org/datasets/global/GLBa0.08_rect/data/ (last access: 17 May 2018).

- Wind velocities were extracted from the Global Data Assimilation System, provided by the Air Resources Laboratory (ARL) of the U.S. National Oceanic and Atmospheric Administration (NOAA). Spatial resolution: $1^{\circ} \times 1^{\circ}$; temporal resolution: $3 \mathrm{~h}$. Further details are available at http://www.ready.noaa.gov/gdas1.php (last access: 17 May 2018); archived data in a proprietary format are available at ftp://arlftp.arlhq.noaa.gov/pub/ archives/gdas1 (last access: 17 May 2018).

- SST data were sourced from a Group for High Resolution Sea Surface Temperature (GHRSST) Level 4 MUR Global Foundation Sea Surface Temperature Analysis, a product developed by the Jet Propulsion Laboratory under NASA MEaSUREs program (JPL, 2015). Spatial resolution: $0.011^{\circ} \times 0.011^{\circ}$; temporal resolution: daily. Detailed information on this data is available at https://mur.jpl.nasa.gov/ (last access: 17 May 2018) and https://podaac.jpl.nasa.gov/ dataset/JPL-L4UHfnd-GLOB-MUR (last access: 17 May 2018).

A direct comparison of the velocity components extracted and interpolated from HYCOM with those of the buoys avail- 


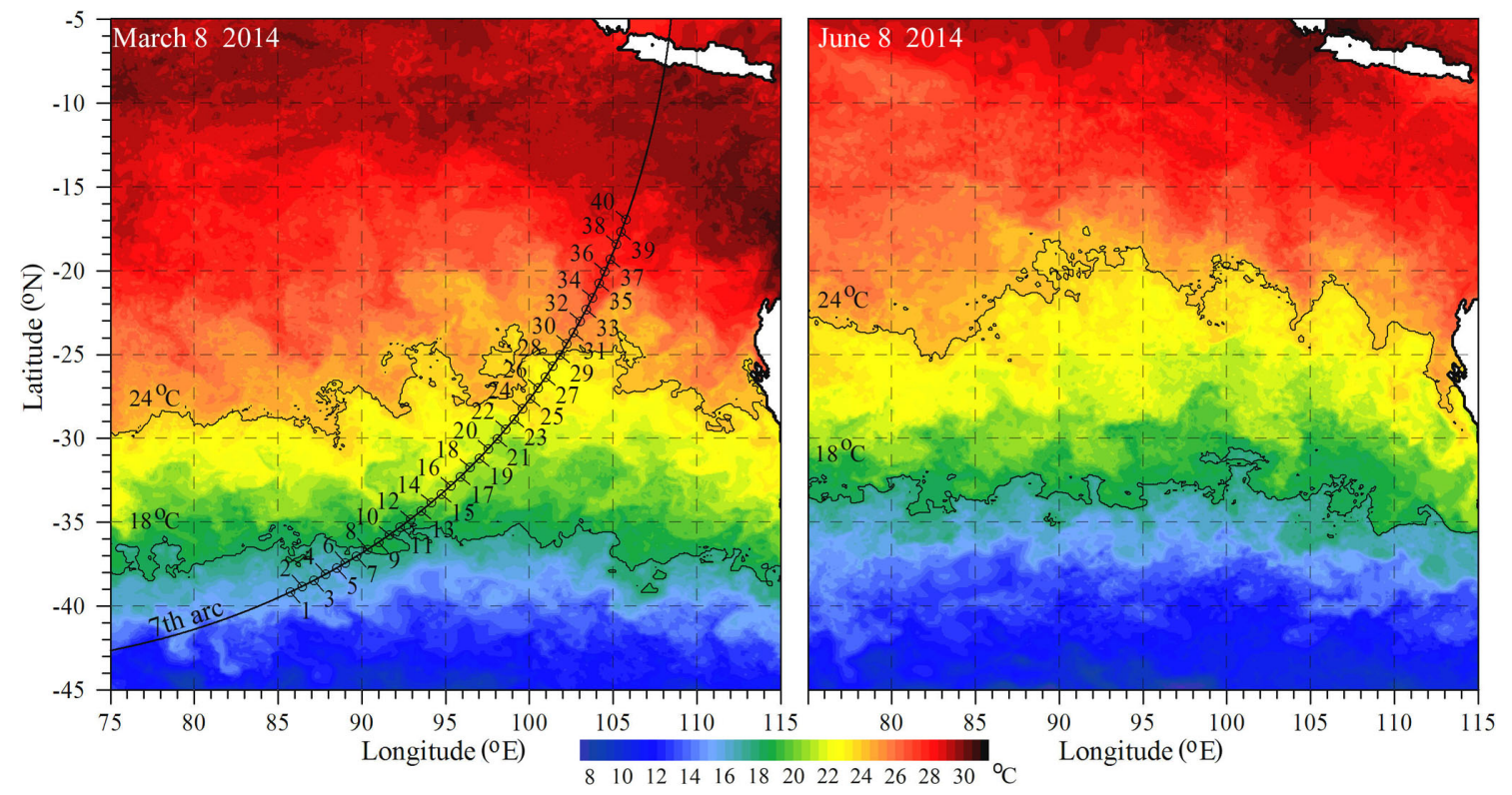

Figure 4. Locations of the selected hypothetical origins on the seventh arc and snapshots of the sea surface temperatures.

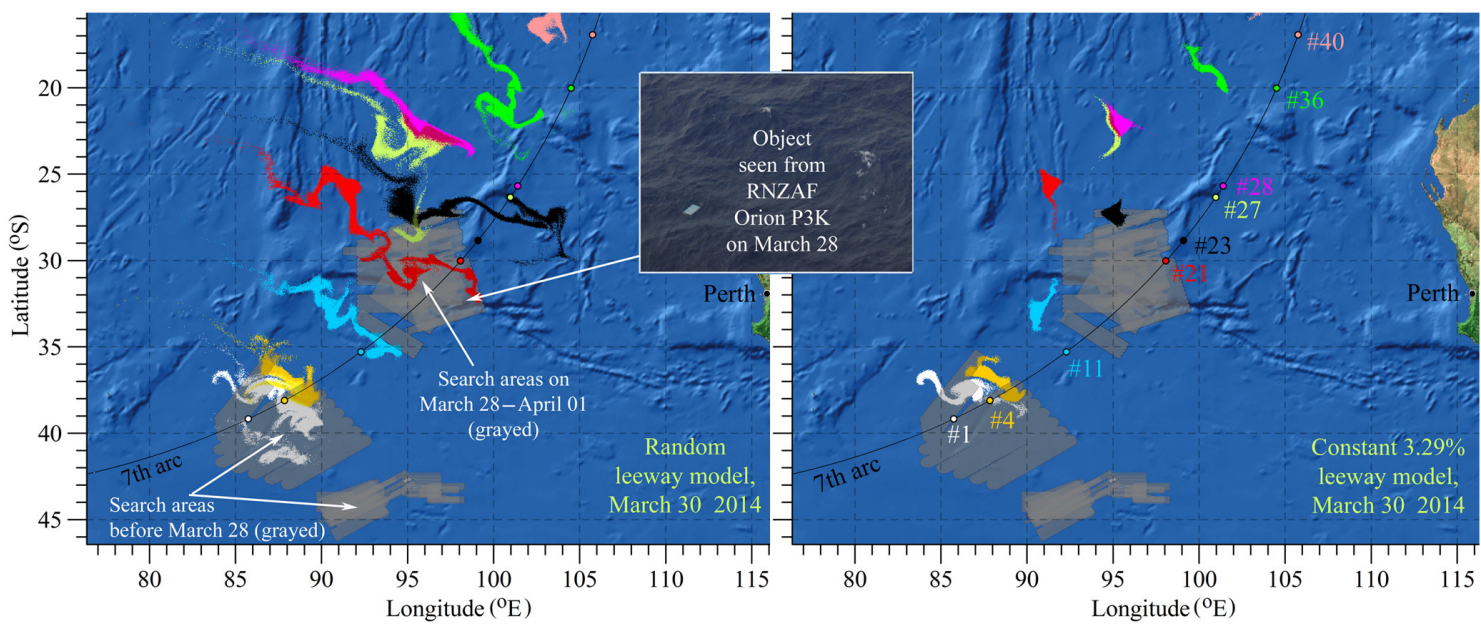

Figure 5. Snapshots of particle ensembles originating from several locations (indicated with corresponding colours of ensembles) on 30 March for two models and the areas surveyed by 2 April. Sources of the aerial search maps and photograph: JAAC (2014), AMSA (2014).

able from the National Oceanic and Atmospheric Administration's Global Drifter Program (Eliot et al., 2016), which passed through the study domain from March 2014 until December 2016 (a total of 820801 samples) have shown rms errors of 26.6 and $26.0 \mathrm{~cm} \mathrm{~s}^{-1}$ for the easterly and northerly components respectively. However, further analysis is required to understand the contribution of wind to these errors (e.g. Griffin et al., 2017, suggest that the average leeway factor of the GDP buoys is around $1.2 \%$ ) and, more importantly, how they can affect modelling accuracy, particularly with regard to whether they are stochastic or systematic.

\section{Results}

\subsection{Efficacy of the aerial search}

An extensive aerial search for MH370 debris, which lasted from 18 March to 27 April 2014, (e.g. JAAC, 2014; AMSA, 2014), failed to find any debris relevant to MH370. Although some suspected objects were observed on 28-31 March (AMSA, 2014), such as a rectangular object photographed from the specialised Royal New Zealand Airforce (RNZAF) Orion P3K survey aircraft (Fig. 5), subsequent attempts to recover them were unsuccessful.

Snapshots of the modelled particle locations in the ensembles originating from nine selected locations along the sev- 

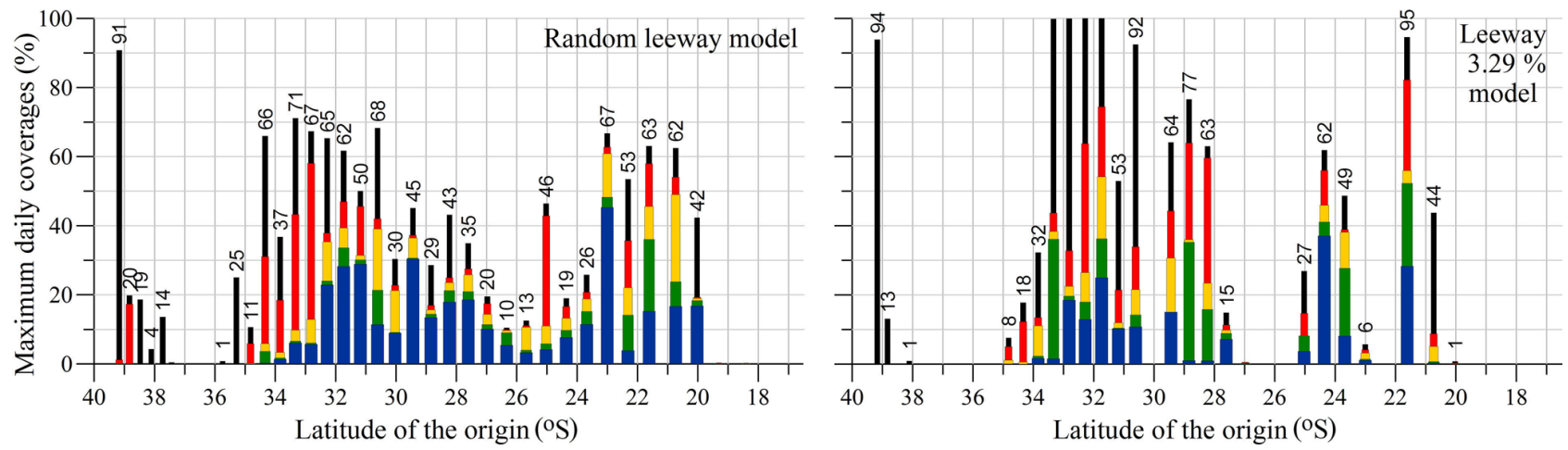

Figure 6. The five largest daily coverages during the aerial search (in descending order: black, red, yellow, green, and blue bars).

Table 2. Estimated maximum daily coverage (\%), respective date of its occurrence (2014), and cumulative coverage (\%) for each screened ensemble based on the proposed random leeway factor model.

\begin{tabular}{|c|c|c|c|c|c|c|c|c|c|c|c|c|c|c|c|}
\hline No. & $\begin{array}{l}\text { Max. } \\
\text { daily }\end{array}$ & $\begin{array}{l}\text { Cumu- } \\
\text { lative }\end{array}$ & Date & No. & $\begin{array}{l}\text { Max. } \\
\text { daily }\end{array}$ & $\begin{array}{l}\text { Cumu- } \\
\text { lative }\end{array}$ & Date & No. & $\begin{array}{l}\text { Max. } \\
\text { daily }\end{array}$ & $\begin{array}{l}\text { Cumu- } \\
\text { lative }\end{array}$ & Date & No. & $\begin{array}{l}\text { Max. } \\
\text { daily }\end{array}$ & $\begin{array}{l}\text { Cumu- } \\
\text { lative }\end{array}$ & Date \\
\hline 1 & 90.7 & 91.9 & Mar 19 & 11 & 24.9 & 25.1 & Apr 01 & 21 & 30.4 & 114.1 & Mar 31 & 31 & 25.8 & 132.9 & Apr 04 \\
\hline 2 & 19.8 & 37.2 & Mar 19 & 12 & 10.7 & 16.9 & Apr 01 & 22 & 45.1 & 279.0 & Mar 28 & 32 & 66.7 & 419.3 & Apr 04 \\
\hline 3 & 18.6 & 18.6 & Mar 18 & 13 & 66.0 & 107.0 & Apr 01 & 23 & 28.6 & 116.1 & Apr 02 & 33 & 53.4 & 137.4 & Apr 05 \\
\hline 4 & 4.3 & 4.3 & Mar 19 & 14 & 36.8 & 62.6 & Apr 01 & 24 & 43.1 & 192.3 & Mar 28 & 34 & 63.1 & 254.8 & Apr 05 \\
\hline 5 & 13.6 & 13.6 & Mar 19 & 15 & 71.1 & 142.0 & Mar 31 & 25 & 34.9 & 199.1 & Mar 28 & 35 & 62.4 & 234.6 & Apr 05 \\
\hline 6 & 0.4 & 0.4 & Mar 19 & 16 & 67.3 & 160.7 & Mar 31 & 26 & 19.5 & 136.2 & Mar 29 & 36 & 42.3 & 138.9 & Apr 03 \\
\hline 7 & $<0.1$ & $<0.1$ & Mar 19 & 17 & 65.3 & 244.3 & Apr 01 & 27 & 10.5 & 68.8 & Apr 20 & 37 & 0.3 & 0.6 & Apr 05 \\
\hline 8 & 0 & 0 & - & 18 & 61.7 & 295.1 & Mar 29 & 28 & 12.5 & 46.3 & Apr 18 & 38 & 0.2 & 0.6 & Apr 05 \\
\hline 9 & $<0.1$ & $<0.1$ & Apr 01 & 19 & 50.0 & 256.8 & Mar 29 & 29 & 46.4 & 126.3 & Apr 13 & 39 & 0 & 0 & - \\
\hline 10 & 0.8 & 0.8 & Apr 01 & 20 & 68.3 & 245.1 & Mar 29 & 30 & 19.0 & 100.8 & Apr 04 & 40 & 0 & 0 & - \\
\hline
\end{tabular}

enth arc on 28 March and 5 April are depicted in Fig. 5 for the random and constant $3.29 \%$ leeway factor models. An animation, which shows computed daily positions of these ensembles and search areas, is presented in the Supplement S1. As seen, the leeway factor had a significant influence on the dispersion, which was notably more intense for the ensembles that comprised particles of various leeway factors. Furthermore, the difference in the leeway factors could presumably explain the failed attempts to track suspected objects with the help of deployed buoys. It is worth noting that the aerial survey appears to be rather inefficient for the objects characterised by the leeway factor of $3.29 \%$ (such as the flaperon), which originated from the locations around $30^{\circ} \mathrm{S}$ or from the segment from 25.5 to $28^{\circ} \mathrm{S}$ of the seventh arc.

To better understand reasons contributing to the aerial search failure, daily efficacies of the search were analysed in terms of the percentages $E_{j, k}$ of particles $i_{j, k}$ of each $j$ th ensemble, which were in the search area $\Omega_{k}$ on the $k$ th day of the search:

$E_{j, k}=\frac{1}{N}\left(\sum_{\Omega_{k}} i_{j, k}\right) \times 100 \%$, where $N=50000$ is the number of particles per ensemble. Respective areas $\Omega_{k}$ were obtained by digitising maps published in AMSA (2014) and JAAC (2014) portals.

As the value of the maximum single-day coverage $\max _{k} E_{j, k}$ could be downplayed by other factors affecting detection capability, such as poor weather conditions, the five largest estimated daily coverages of particle ensembles are presented in Fig. 6 for the random and constant $3.29 \%$ leeway factor models versus the origin's latitude along the seventh arc. The maximum single-day coverage, date of its occurrence, and cumulative coverage over the entire duration of the search campaign are summarised in Table 2 for each screened ensemble based on the proposed random leeway factor model. The cumulative coverages were defined as the sum $\sum_{k} E_{j, k}$ of daily ensemble coverages over the duration of the search campaign. It can be viewed as an additional selection criterion of a more likely origin when peak daily coverages are similar, such as in the case of ensembles no. 21 and no. 25 (Table 2).

As seen, if the crash site was located between 30.5 and $34.5^{\circ} \mathrm{S}$ or between 20 and $25^{\circ} \mathrm{S}$, locations for fragments of 9M-MRO would have been relatively well covered, which would consequently increase the chances of their detection. The coverage of MH370 debris would have been notably 
lower if the crash site was located between 25.5 and $27.5^{\circ} \mathrm{S}$. In particular, for the origins no. 27 and no. 28 , the maximum coverages of approximately $10 \%$ occurred more than 6 weeks after the crash, which is a rather low figure bearing in mind that decay and sinking processes were not taken into consideration. A relatively poor coverage is also noted for the origins no. 21 and no. 23.

Interestingly, a rectangular object spotted from RNZAF Orion P3K on 28 March was in the area, where some debris originating from the location no. $21\left(98.07^{\circ} \mathrm{E}, 30.03^{\circ} \mathrm{S}\right)$ could be expected according to the random leeway model (Fig. 5). Furthermore, the timing of the peak coverages (2831 March; see Table 2) for the debris originating from this and neighbouring locations is consistent with the dates when a number of floating objects were detected there.

\subsection{SST along the path of the flaperon}

One of the major goals of this study is to address a question whether the ambient water temperatures along modelled particle tracks could match the temperature variation derived from the biochemical analysis of the barnacles attached to the flaperon (De Deckker, 2017) and, if yes, whether this information could help to further refine the search area. Therefore, those particles were of interest which satisfied the following two conditions:

- A particle must have arrived at the St-André beach, a place where the flaperon was found (assumed coordinates $55.67^{\circ} \mathrm{E}, 20.93^{\circ} \mathrm{S}$ ), by 29 July 2015 .

- The ambient SST at a particle's location should have first exceeded $24^{\circ} \mathrm{C}$, then dropped down below $18^{\circ} \mathrm{C}$, and then risen up again to $25^{\circ} \mathrm{C}$ or higher.

Due to the inherent uncertainty in the temperature estimations based on the barnacle analysis, a score-based function $S$ was introduced to identify those particles which approximately satisfied the two aforementioned conditions:

$S_{i}=S_{i, d} \quad S_{i, \theta}$

Here the term $S_{i, d}=\exp \left(-0.5 d_{i}^{2} / d_{\text {ref }}^{2}\right)$ is responsible for the first condition above; $d_{i}=d_{i}\left(\psi_{i}, \varphi_{i}\right)$ is the ground distance between the $i$ th particle location on 29 July 2015 and the St-André beach; $d_{\text {ref }}=50 \mathrm{~km}$ is the reference distance, which was chosen to be the approximate linear dimension of La Réunion. The term $S_{i, \theta}$ responsible for the second condition, was formulated in the following way:

$$
S_{i, \theta}=\left\{\begin{array}{cl}
\frac{S_{i, \theta_{1}}+S_{i, \theta_{2}}+S_{i, \theta_{3}},}{3}, & \text { if } \exists\left\{t_{1}, t_{2}, t_{3}\right\} \in\left[T_{s}, T_{e}\right]: \\
& \max \theta_{i}(t) \geq 23^{\circ} C, \\
& 0 \leq t \leq t_{1} \\
& \min _{t_{1} \leq t \leq t_{2}} \theta_{i}(t) \leq 19^{\circ} C, \\
& \max \theta_{i}(t) \geq 24^{\circ} C, \\
& t_{2} \leq t \leq T \\
& \text { otherwise, }
\end{array}\right.
$$

where $\theta_{i}(t)$ is the SST at the $i$ th particle location at the time $t\left(T_{s} \leq t \leq T_{e}\right.$, where $T_{s}$ and $T_{e}$ correspond to 8 March 2014 and 29 July 2015 respectively) and

$$
\begin{aligned}
& S_{i, \theta_{1}}=\min \left(\max \left(\max _{0 \leq t \leq t_{1}} \theta_{i}(t)-23,0\right), 1\right), \\
& S_{i, \theta_{2}}=\min \left(\max \left(19-\min _{t_{1} \leq t \leq t_{2}} \theta_{i}(t), 0\right), 1\right), \\
& S_{i, \theta_{3}}=\min \left(\max \left(\max _{t_{2} \leq t \leq T} \theta_{i}(t)-24,0\right), 1\right) \text {. }
\end{aligned}
$$

The purpose of such a formulation Eq. (20) is to relax the selection criteria and avoid discontinuities by assigning a positive score to a particle even if it did not arrive precisely at the St-André beach or if it did not strictly satisfy the temperature condition (the tolerance allowing for a positive score was set to $1^{\circ} \mathrm{C}$ ). As a result, the maximum score a particle could receive was 1 . If a particle arrived at La Réunion before 29 July, it was still assigned a positive score. If a particle was located at a distance greater than $152 \mathrm{~km}$ from St-André beach on 29 July, it could not receive a score higher than 0.01 . If the SST at a particle's location never reached $23^{\circ} \mathrm{C}$ or never dropped below $19^{\circ} \mathrm{C}$ after that, such a particle received a 0 score.

The percentages of particles in ensembles which received scores $S>0.01$ vs. origin's latitude along the seventh arc are shown in Fig. 7 for all the four model set-ups, together with the normal distribution fitting. As seen, for the leeway factors and non-zero drift angles of the flaperon determined by the DGA (Daniel, 2016), the segment centred at $28.2 \pm 3^{\circ}$ appears to be the most likely area where the flaperon began its journey. A fraction of particles in the two other models (characterised by zero drift angle), which satisfied the two conditions, could reach a surprisingly high value of $1 \%$; however, both the random leeway factor and constant $3.29 \%$ leeway factor models indicated the peak fitted probabilities at 30.8 and $31.9^{\circ} \mathrm{S}$ respectively. The screened origins south of $36.5^{\circ} \mathrm{S}$ or north of $20^{\circ} \mathrm{S}$ are deemed to be unlikely starting locations of the flaperon, as none of the four models predicted a notable percentage of particles meeting the two requirements above for the corresponding ensembles.

Examples of the first six particle tracks, which received the highest scores $S$, are depicted in Fig. 8 for the scenario corresponding to the leeway factor of $3.29 \%$ and drift angle $18^{\circ}$ to the left, starting from the origin no. $23\left(99.09^{\circ} \mathrm{E}, 28.84^{\circ} \mathrm{S}\right)$. All these particles received the scores $S_{i}>0.83$, and they arrived at La Réunion between 17 June (black track) and 17 July (yellow track) 2015. As seen, the two main reasons for the drop in the ambient water temperature from $23-25^{\circ} \mathrm{C}$ to as low as $16^{\circ} \mathrm{C}$ are as follows:

- seasonal cooling of the water surface (see comparison of the SSTs on 8 March and 8 June 2014 in Fig. 4);

- entrapment in counter-clockwise eddies, which could first carry the flaperon northwestward up to $22-25^{\circ} \mathrm{S}$ latitudes and then southward to $30-33^{\circ} \mathrm{S}$ (Fig. 8). 

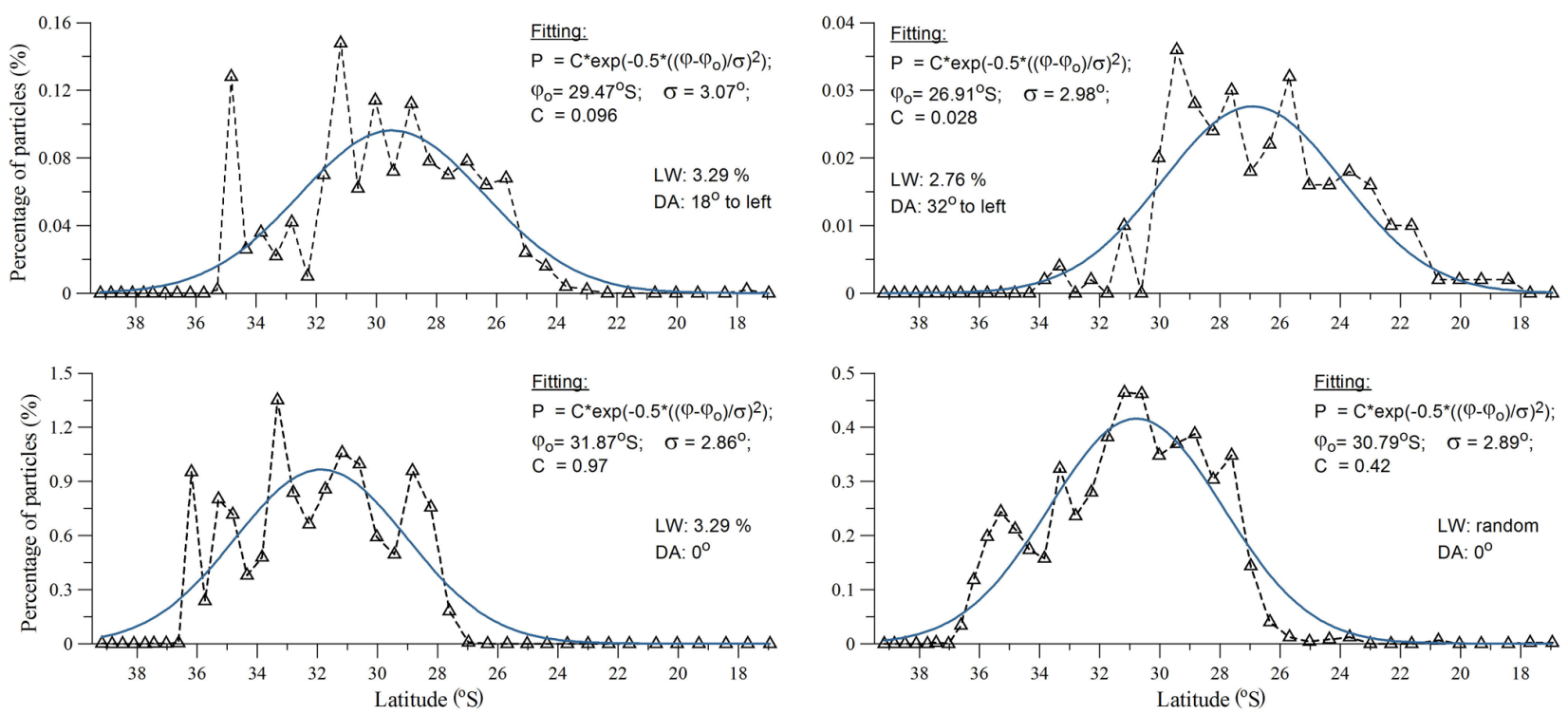

Figure 7. Percentages of particles satisfying distance and temperature criterion with the score $S>0.01$ (LW: leeway factor; DA: drift angle).

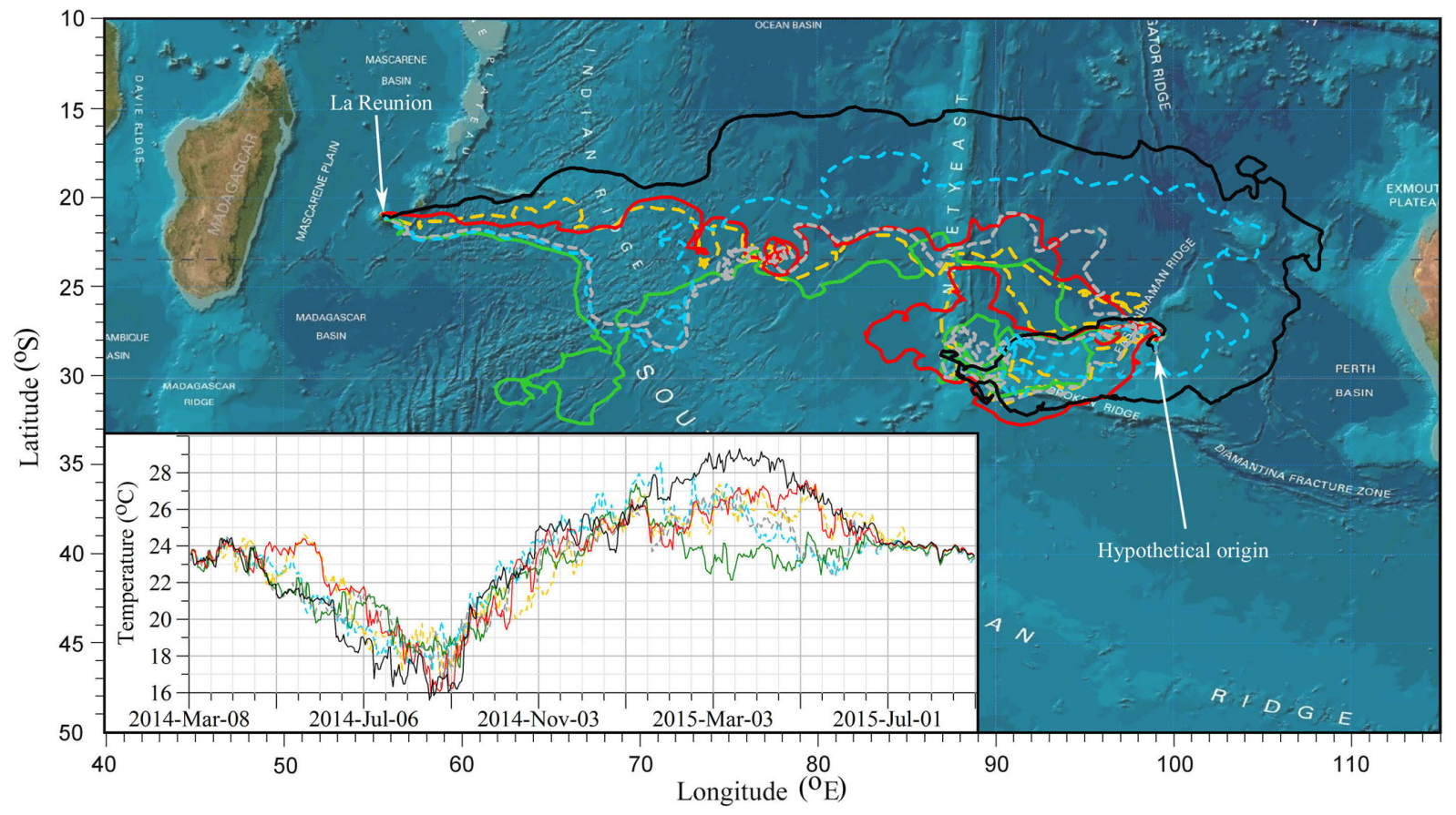

Figure 8. Sample tracks and temperatures for the six particles of ensemble no. 23 (leeway $3.29 \%$; drift angle $18^{\circ}$ ).

\subsection{Beached debris distribution}

A total of 27 possibly relevant and confirmed fragments of 9M-MRO were recovered in La Réunion, Mauritius, Rodrigues Island, Madagascar, Mozambique, South Africa, and Tanzania as of April 2017 according to the MSIT (2017); none was ever found in Australia, although a suspected object, the unopened towelette bearing the MAS logotype, was discovered at Thirsty Point. The distribution of the debris found offers a useful insight into the possible location of the crash site (e.g. Jansen et al., 2016; Pattiaratchi and Wijeratne, 2016; Griffin et al., 2017), although no consensus has been reached to date with regard to the origin. Similarly to the aforementioned studies, an effort was made in this study to analyse the spatial distribution of the fragments washed ashore.

A sample snapshot of the computed particle locations on 31 December 2016 is depicted in Fig. 9 for the random lee- 


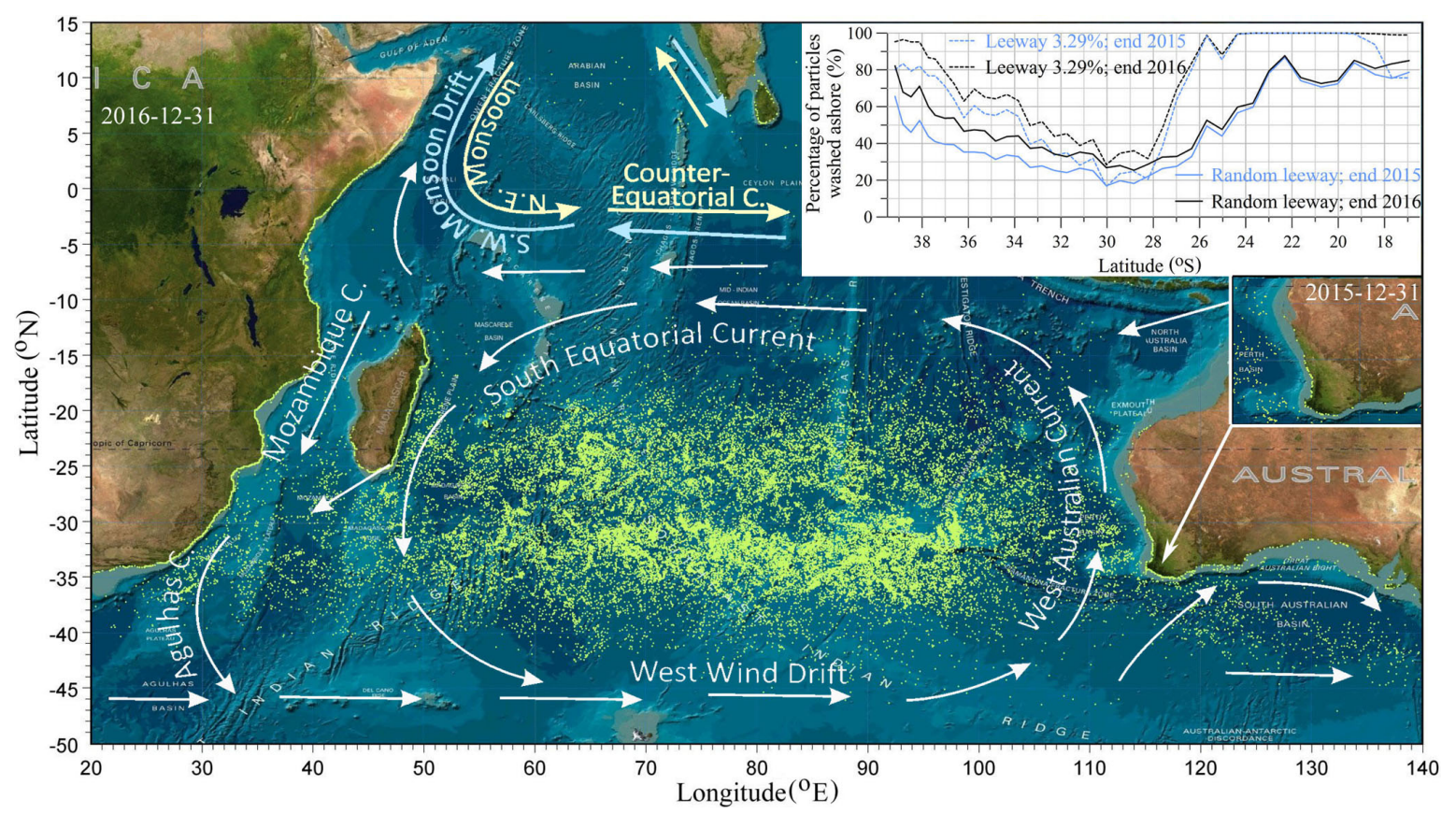

Figure 9. Snapshot of particle locations on 31 December 2016 (random leeway model; origin no. 23) and percentages of particles washed ashore.

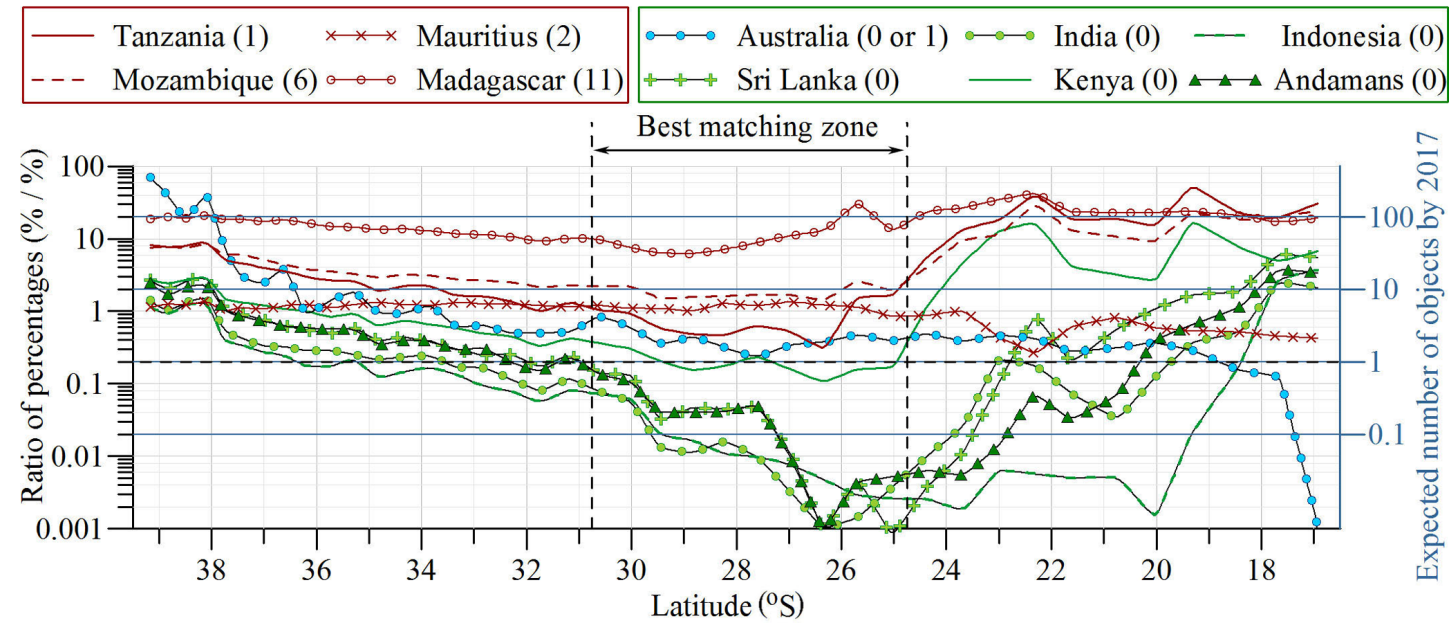

Figure 10. Expected number of objects to be found in several countries by 31 December 2016 vs. origin's latitude.

way model, origin no. 23. Corresponding animation S2a is included in the Supplement. Considerable beaching of particles of this ensemble was modelled in Africa, Madagascar, La Réunion, and Mauritius, but it was negligible in Australia. The total fractions of particles landed by the end of 2015 and 2016 are shown in the top right corner for all the screened origins, both the random and constant $3.29 \%$ leeway factor models. This result is, in part, due to the West Australian Current, which entrains large percentages of particles from the northern and southern origins (see animations S3 and S4), while most of particles from the middle of the screened seg- ment of the seventh arc remain trapped in the Indian Ocean Gyre.

An interesting result can be obtained by comparing the modelled ratios of the fractions of particles washed ashore in several countries to the ratios of the number of fragments actually recovered in these countries. Such a comparison provides an indication of a number of fragments expected to be found by a certain date assuming the same reporting factors. Figure 10 shows computed ratios for the random leeway model, using South Africa as the reference (a total of five objects were found there). As seen, more than five objects could be expected in Australia, at least two objects in Sri Lanka, 


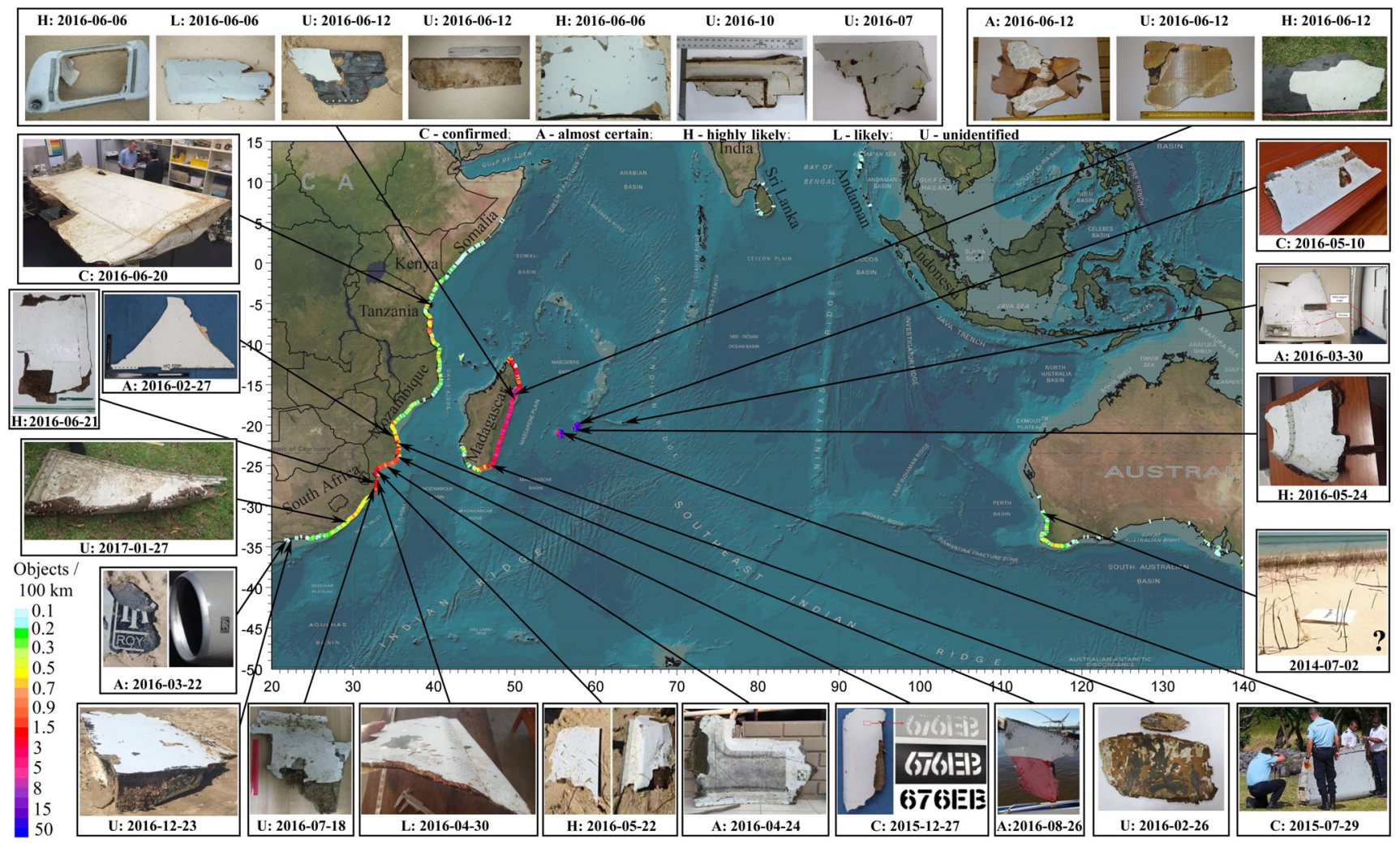

Figure 11. Expected along-shore concentration of beached MH370 fragments from the hypothetical origin no. 23 to be found by the end of 2016.

Table 3. Sensitivity of the percentages of particles washed ashore by the end of 2016 to the number of particles in ensembles (origin no. 23).

\begin{tabular}{|c|c|c|c|c|c|c|c|c|c|c|c|c|c|c|c|}
\hline 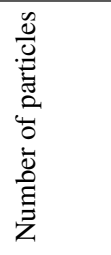 & 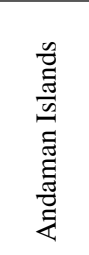 & 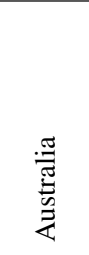 & 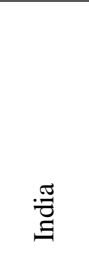 & 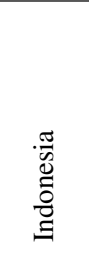 & 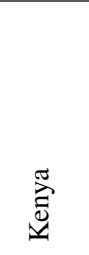 & 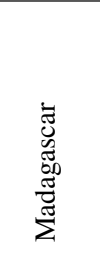 & 鸹 & 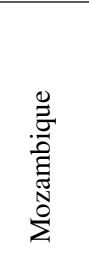 & 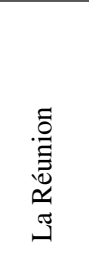 & 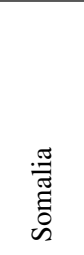 & 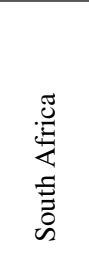 & $\begin{array}{l}\frac{\tilde{y}}{\tilde{E}} \\
\tilde{\Xi} \\
\vec{E}\end{array}$ & 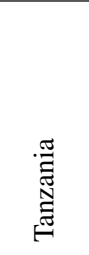 & 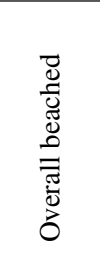 & 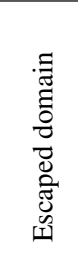 \\
\hline 50000 & 0.078 & 0.854 & 0.022 & 0.048 & 0.300 & 12.166 & 1.966 & 3.068 & 2.338 & 0.272 & 1.946 & 0.090 & 0.952 & 25.430 & 0.724 \\
\hline 500000 & 0.090 & 0.866 & 0.037 & 0.037 & 0.329 & 12.163 & 1.976 & 3.097 & 2.438 & 0.289 & 1.908 & 0.087 & 0.977 & 25.703 & 0.744 \\
\hline
\end{tabular}

nine objects in Tanzania, and three objects in Kenya, should the origin be south of $35^{\circ} \mathrm{S}$. At least one fragment could be expected in Sri Lanka and several in Kenya and Tanzania for the origins north of $23^{\circ} \mathrm{S}$. The best matching segment of the seventh arc is located approximately between 26.5 and $31^{\circ} \mathrm{S}$. In particular, the ratio of the percentages of particles beached in Mozambique to those in South Africa was in the range of 1.4 to 1.7 , being in a good agreement with the actual ratio of $6: 5$.

To estimate the along-shore concentration of MH370 objects expected to be found by a certain time, the twodimensional Gaussian smoothing filter was first applied to obtain a smoothed concentration of beached particles:
$P(\psi, \varphi)=\frac{1}{2 \pi d_{\mathrm{ref}}^{2}} \sum_{i=1}^{M} \exp \left(-\frac{d_{i}^{2}\left(\psi, \varphi, \psi_{i}, \varphi_{i}\right)}{2 d_{\mathrm{ref}}^{2}}\right)$,

where $d_{i}$ is the ground distance between the $i$ th beached particle and the location of interest $(\psi, \varphi), d_{\text {ref }}=5 \mathrm{~km}$ is the size of the smoothing filter, and $M$ is the number of beached particles. To compute the along-shore density distribution of objects expected to be found, this function was numerically integrated over a relatively narrow band-shaped area $\Omega_{i}$ long-wise centred at the shorelines, divided by the respective lengths of the shoreline segments $\Delta s_{i}$, and prorated by the ratio of the number of fragments found in South Africa 

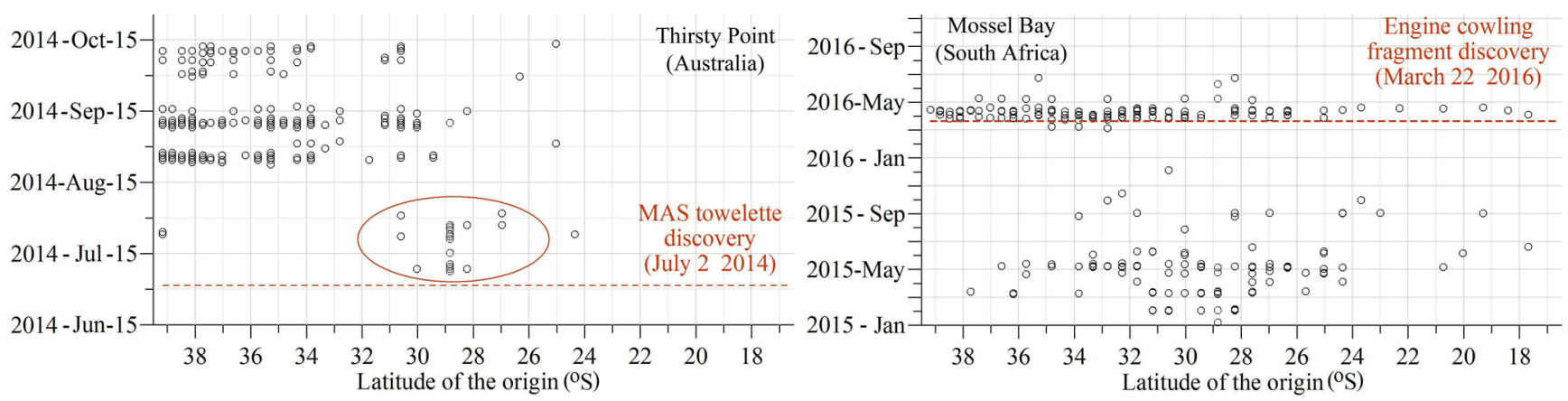

Figure 12. Arrival times of particles to the proximity of Thirsty Point (Australia) during 2014 and Mossel Bay (South Africa).

$N_{\mathrm{SA}}=5$ to the number of particles landed in South Africa $M_{\mathrm{SA}}$ :

$\frac{\mathrm{d} N}{\mathrm{~d} s} \approx \frac{N_{\mathrm{SA}}}{M_{\mathrm{SA}}} \frac{1}{\Delta s_{i}} \iint_{\Omega_{i}} P(\psi, \varphi) \mathrm{d} \Omega$.

An example of the concentration of objects estimated in this way is shown in Fig. 11 for the ensemble released from origin no. 23 (random leeway model); concentrations for all the other studied origins are presented in Fig. S5 and animation S5. As seen, the locations of the elevated concentrations are in a fairly good agreement with the locations where the fragments were found, except Rodrigues Island, which was not properly resolved by HYCOM. More fragments could be expected in Tanzania at 7.0 and $8.2^{\circ} \mathrm{S}$.

Screening of origin no. 23 has revealed that the elevated concentration near Cape Leeuwin in Australia is due to the beaching which mainly occurred in 2016. During 2014-2015 notable arrival of particles from this origin to Australia took place only around Windy Harbour and Thirsty Point, where the unopened MAS towelette was found on 2 July 2014 (assumed coordinates $115.3^{\circ} \mathrm{E}, 31^{\circ} \mathrm{S}$ ). A detailed analysis has shown that 8 particles of the respective ensemble landed near Thirsty Point during 8-11 July, and 10 more particles arrived before 28 July. All the first 8 particles were characterised by relatively low leeway factors ranging from 0.1 to $0.4 \%$. This suggests a systematic feature rather than a random occurrence. Systematic arrival of particles from the origins north of $27^{\circ} \mathrm{S}$ or south of $31^{\circ} \mathrm{S}$ was not predicted earlier than in the last week of July, as seen in Fig. 12.

Figure 12 also shows the arrival times of particles to Mossel Bay in South Africa, where the engine cowling fragment was found. Being the fourth object found, it had covered a long distance over a relatively short time interval. The modelling has shown the possibility of the sporadic arrival of high-windage particles to Mossel Bay as early as February 2015, but systematic arrival was predicted only for MarchMay 2016, consistent with the discovery date.

To understand the sensitivity of the results presented in this section to the number of particles in ensembles, a nu- merical experiment was conducted using 500000 particles for origin no. 23 in the random-leeway factor model (see animation S2b). The results of this experiment (Table 3) indicate that the use of 50000 particles is sufficient to obtain fairly reliable statistics, and a further increase in the number of particles would unlikely be beneficial.

\section{Conclusions}

The drift study of MH370 debris was conducted by means of numerical modelling using a forward particle tracking technique. A total of 40 hypothetical locations of the crash site along the seventh arc were screened. Three major aspects were considered: (1) the efficacy of the aerial search; (2) ambient water temperatures along the path of the flaperon to La Réunion; (3) the spatial distribution of the debris washed ashore.

The governing equations were numerically integrated in the geocentric Cartesian coordinate system, where the Earth surface was approximated by the WGS 84 ellipsoid. Four models with respect to the leeway factors and drift angles were considered, including a proposed model of random distribution of the leeway factors of particles in an ensemble.

The obtained results indicate the significance of the leeway factor in all the three aspects considered. In addition to the uncertainties in the model forcing, assumptions, and simplification, a judgment about the most likely location of the crash site depends on weights assigned to the aerial search, the accuracy of the barnacle biochemical analysis, and the probability of fragments not only being washed ashore but also being recovered and reported. While it does not appear to be possible to confidently point out the location of the crash site based on the drift study alone, a few observations can be made with regard to various segments of the seventh arc:

- South of $36^{\circ}$ S. Considerable beaching where no fragments were found, particularly in Australia and Sri Lanka; incompatibility with the water temperatures suggested by the barnacle biochemical analysis. 
- 34.5 to $36^{\circ} \mathrm{S}$. While corresponding areas were poorly surveyed during the aerial search, considerable beaching could be expected in several countries where no fragments were found, particularly in Australia.

- 30.5 to $34.5^{\circ} \mathrm{S}$. Excellent aerial coverage of the debris cloud originating from this segment makes the crash site unlikely to be located within it.

- 25.5 to $30.5^{\circ} \mathrm{S}$. Consistency with the barnacle temperature analysis; elevated concentration of beached particles, where the fragments of 9M-MRO were found; several "gaps" in the aerial search; floating objects detected on 28-31 March; possible consistency with the early arrival of the MAS towelette at Thirsty Point.

- North of $25.5^{\circ} \mathrm{S}$. Inconsistency with the distribution of the debris washed ashore; incompatibility with the barnacle temperature analysis; good aerial coverage of the areas corresponding to the origins from 20 to $25^{\circ} \mathrm{S}$.

Summarising all the above, the most likely area of the crash site appears to be between 25.5 and $30.5^{\circ} \mathrm{S}$, with the segment from 28 to $30^{\circ} \mathrm{S}$ being the most promising. This area is consistent with the original definition of a highpriority search zone by the ATSB in June 2014.

Data availability. All the underlying data used in this paper are publicly available. In particular, the third-party sources of global current, wind, and SST data are detailed in Sect. 2.2.2. Raw particle track data modelled in this study are currently not available online due to the large volume of data.

\section{The Supplement related to this article is available online at https://doi.org/10.5194/os-14-387-2018-supplement.}

Competing interests. This study was conducted solely at the author's expense. The author declares that he has no conflict of interest.

Acknowledgements. Use was made of current data from HYCOM, provided by the National Ocean Partnership Program and the Office of Naval Research; the wind data were sourced from NOAA ARL GDAS; the SST data were obtained from NASA EOSDIS PODAAC at the JPL. The author is thankful to the two anonymous reviewers and the topic editor, who helped to improve the clarity and content of this paper.

Edited by: John M. Huthnance

Reviewed by: two anonymous referees

\section{References}

Al-Rabeh, A. H., Lardner, R. W., and Gunay, N.: Gulfspill Version 2.0: a software package for oil spills in the Arabian Gulf, Environ. Modell. Softw., 15, 425-442, 2000.

Australian Maritime Safety Authority: MH370 Search - Media kit, available at: https://www.amsa.gov.au (last access: 2 May 2017), 2014.

Ashton, C., Bruce, A. S., Colledge, G., and Dickinson, M.: The search for MH370, J. Navigation, 68, 1-22, 2015.

Australian Transport Safety Bureau: MH370 - Definition of Underwater Search Areas, 26 June 2014, available at: http://www.atsb. gov.au (last access: 17 May 2017), 2014a.

Australian Transport Safety Bureau: MH370 - Flight Path Analysis Update, 30 July 2014, available at: http://www.atsb.gov.au (last access: 17 May 2017), 2014b.

Australian Transport Safety Bureau: MH370 - Definition of Underwater Search Areas, 3 December 2015, available at: http: //www.atsb.gov.au (last access: 14 May 2017), 2015.

Blumberg, A. F. and Mellor, G. L.: A description of a threedimensional coastal ocean circulation model, Three-Dimensional Coastal Ocean Models, edited by: Heaps, N., Washington D.C., Am. Geoph. Union, 1-16, 1987.

Box, G. E. P. and Muller, M. E.: A Note on the Generation of Random Normal Deviates, Ann. Math. Stat., 29, 610-611, 1958.

Breivik, Ø., Allen, A. A., Maisondieu, C., and Roth, J. C.: Windinduced drift of objects at sea: The leeway field method, Appl Ocean Res., 33, 100-109, 2011.

Chassignet, E. P., Hurlburt, H. E., Smedstad, O. M., Halliwell, G. R., Hogan, P. J., Wallcraft, A. J., Baraille, R., and Bleck, R.: The HYCOM (HYbrid Coordinate Ocean Model) data assimilative system, J. Mar. Syst., 65, 60-83, 2007.

Daniel, P.: Rapport d'étude, Dérive à rebours de flaperon, Météo France, 8 Février 2016, Direction des Opérations pour la Prévision, Département Prévision marine et Océanographique, 32 pp., 2016 (in French).

Daniel, P., Gwénaële, J., Cabioc'h, F., Landau, Y., and Loiseau, E.: Drift modeling of cargo containers, Spill Sci. Technol. B., 7, 279-288, 2002.

Davey, S., Gordon, N., Holland, I., Rutten, M., and Williams, J.: Bayesian Methods in the Search for MH370, Springer Briefs in Electrical and Computer Engineering, 114 pp., 2016.

De Deckker, P.: Chemical investigations on barnacles found attached to debris from the MH370 aircraft found in the Indian Ocean, Appendix F in ATSB report: "The Operational Search for MH370", AE-2014-054, 3 October 2017, available at: http: //www.atsb.gov.au, last access: 3 October 2017.

DHI Water and Environment: MIKE21 and MIKE3 Flow Model FM, Particle Tracking Module, Scientific Documentation, 28 pp., Hörsholm, Denmark, available at: www.dhigroup.com, last access: 16 March 2009.

Durgadoo, J. and Biastoch, A.: Where is MH370? GEOMAR Helmholtz Centre for Ocean Research Kiel, 28 August 2015, available at: http://www.geomar.de (last access: 17 May 2018), 2015.

Eisenberg, B. and Sullivan, R.: Why is the Sum of Independent Normal Random Variables Normal, Math. Mag., 81, 362-366, 2008.

Elipot, S., Lumpkin, R., Perez, R. C., Lilly, J. M., Early, J. J., and Sykulski, A. M.: A global surface drifter dataset at 
hourly resolution, J. Geophys. Res.-Oceans, 121, 2937-2966, https://doi.org/10.1002/2016JC011716, 2016.

Gandin, A. S., Laightman, D. L., Matveyev, L. T., and Yudin, M. I.: Fundamentals of dynamic meteorology, Gidrometizdat, Leningrad, 647 pp., 1955 (in Russian).

García-Garrido, V. J., Mancho, A. M., Wiggins, S., and Mendoza, C.: A dynamical systems approach to the surface search for debris associated with the disappearance of flight MH370, Nonlin. Processes Geophys., 22, 701-712, https://doi.org/10.5194/npg22-701-2015, 2015.

Griffin, D., Oke, P., and Jones, E.: The search for MH370 and ocean surface drift - Part II, CSIRO Oceans and Atmosphere, Australia, Report no. EP172633, prepared for the Australian Transport Safety Bureau, 13 April 2017, available at: https://www.atsb. gov.au, last access: 13 May 2017.

Jansen, E., Coppini, G., and Pinardi, N.: Drift simulation of MH370 debris using superensemble techniques, Nat. Hazards Earth Syst. Sci., 16, 1623-1628, https://doi.org/10.5194/nhess16-1623-2016, 2016.

Joint Agency Coordination Centre (JACC): Media Releases - 2014, available at: http://jacc.gov.au/media/releases/2014/april (last access: 2 May 2017), 2014.

JPL MUR MEaSUREs Project: GHRSST Level 4 MUR Global Foundation Sea Surface Temperature Analysis (v4.1), Ver. 4.1. PO.DAAC, CA, USA, available at: https://podaac.jpl.nasa.gov/ dataset/MUR-JPL-L4-GLOB-v4.1 (last access: 17 May 2018), 2015.

Korn, G. A. and Korn, T. M.: Mathematical Handbook for Scientists and Engineers, Definitions, Theorems and Formulas for Reference and Reviews, Second Enlarged and Revised Edition, McGraw-Hill Book Company, 832 pp., 1968.

Kraus, E. B.: Atmosphere-Ocean Interaction, edited by: Shepard, P. A., Clarendon Press, Oxford, 1972.

Malaysian ICAO Annex 13: Safety Investigation Team for MH370, Ministry of Transport, Malaysia, Summary of Possible MH370 Debris Recovered, 28 February 2017, available at: http://www. mh370.gov.my, last access: 13 May 2017.
Marsaglia, G. and Zaman, A.: Toward a Universal Random Number Generator, Florida State University Report: FSU-SCRI-8750, 1987.

Maximenko, N., Hafner, J., Speidel, J., and Wang, K. L.: IPRC Ocean Drift Model Simulates MH370 Crash Site and Flow Paths, International Pacific Research Center, School of Ocean and Earth Science and Technology at the University of Hawaii, 4 August 2015, available at: http://iprc.soest.hawaii.edu/news/ MH370_debris/IPRC_MH370_News.php (last access: 17 May 2018), 2015.

Ministry of Transport Malaysia: Official Portal for MH370 Air Incident Investigation, available at: http://www.mot.gov.my/en/ aviation/air-incident-investigation/MH370 (last access: 17 May 2018), 2017.

Pattiaratchi, C. and Wijeratne, S.: Ocean currents suggest where we should be looking for missing flight MH370, The Conversation, 28 July 2016, available at: https://theconversation.com/oceancurrents-suggest-where-we-should-be-looking-for (last access: 17 May 2018), 2016.

Smagorinsky, J.: General circulation experiments with primitive equations, The basic experiment, Mon. Weather Rev., 91, 99164, 1963.

Trinanes, J. A., Olascoaga, M. J., Goni, G. G., Maximenko, N. A., Griffin, D. A., and Hafner, J.: Analysis of flight MH370 potential debris trajectories using ocean observations and numerical model results, J. Oper. Oceanogr., 9, 126-138, 2016.

van Ormondt, M. and Baart, F.: Aircraft debris MH370 makes Northern part of the search area more likely, Deltares News, 31 July 2015, available at: https://www.deltares.nl/en/news/aircraftdebris-mh370-makes-northern-part-of-the-search (last access: 17 May 2018), 2015. 